\title{
Scattering on compact manifolds with infinitely thin horns
}

\author{
Jochen BRÜNING and Vladimir GEYLER
}

\section{Introduction}

In the paper [25] L. D. Faddeev initiated the investigation of the quantum mechanical scattering on manifolds of constant negative curvature with cusps (sometimes also called "horns" [38]); further developments of this theory are presented e.g. in [20], [26], [53], [68]. It is interesting to note that an explicit expression for the reflection coefficient in the case of one horn was obtained earlier by R. Godement [34]. Note also that M. G. Gutzwiller has revealed a relation between the scattering theory on manifolds with horns and the description of chaotic behavior of quantum systems [38], [39], [40].

If we imagine the width of the horns tending to zero, then we obtain a so-called hedgehog-shaped topological space (or "horned manifold"). Strictly speaking, we consider the limit of a family of horned spaces in the sense of the Hausdorff-Gromov distance [36]. The simplest specimen of such a manifold is the Euclidean plane with an attached half-line. The quantum mechanical scattering in this system has been investigated for the first time by P. Exner and P. Šeba [21]; in [23] these authors consider a compact plane domain with a half-line glued to it. A series of significant physical applications of the corresponding results as well as an intensive bibliography related to the subject in question may be found in [24]; we may add that the considered problem is also connected with the scattering on graphs [1], [22], [27], [49], [59]. An explicit expression for the transmission coefficient in the case of two half-lines ("wires") attached to a compact Riemannian manifold of dimension two or three with some special boundary conditions at the points of gluing has been obtained by A. Kiselev [46]. A general method of solving the transmission problem through an arbitrary quantum device was proposed in [32], this method is based on an approach to modeling of quantum systems developed by B. S. Pavlov [60]; some of its applications are given e.g. in [6], [29], 33], [61], 662]. Many-terminal problems for a bounded domain in $\mathbb{R}^{d}(d=2$ or 3$)$ with wires attached to the boundary of the domain are considered recently in [55], [56].

In this paper we consider the quantum mechanical scattering in a hedgehog-shaped space which is constructed by gluing a finite number of half-lines to distinct points of a compact Riemannian manifold of dimension less than four. The Hamiltonian of a quantum 
particle in such a system coincides with a Schrödinger operator on the punctured manifold (the points of gluing are removed) and with the free Schrödinger operator on each half-line. At the gluing points, some boundary conditions are imposed. In particular, the Schrödinger operator in a magnetic field is included in our scheme. The approach we use is based on the Krein resolvent formula from operator extension theory [50], therefore in Sec. 1 we give a very brief sketch of results needed from this theory. Sec. 2 is devoted to the construction of Schrödinger operators on the hedgehog-shaped space; we use the theory of boundary value spaces 35] to describe all possible kinds of boundary conditions defining the Schrödinger operators. We distinguish among them operators of "Dirichlet" and of "Neumann" type. It is worth noting that the results of Sec. 2 are valid for all Riemannian manifolds of dimension less than four, not only for the compact ones. In principle, the definition of the Schrödinger operator on a hedgehog-shaped space may be given in the framework of pseudo-differential operator theory on such a space [66], but our approach is more convenient for investigating the scattering parameters and connected with the approach to spectral problems for point perturbations on Riemannian manifolds [8], [9].

The main results of the paper are contained in Sections 3 and 4 . Here we get a complete description of the spectral structure of Schrödinger operators on hedgehog-shaped spaces (Theorem 4), the proof of existence and uniqueness of scattering states (Theorem 5), and the proof of the unitary nature of the scattering matrix (Theorem 6). An explicit form for the scattering matrix is given in the cases of arbitrary Schrödinger operator on the hedgehog shaped space (Formula (67)). In the particular case of the boundary conditions of Neumann types our formulas contain the result of [46] as a very special case. Theorem 7 from Sec. 4 shows that the positive part of the spectrum of the initial Schrödinger operator on the compact manifold as well as the spectrum of a point perturbation of such an operator may be recovered from the scattering amplitude for one attached half-line (so, an "infinitely thin horn" may be considered as a kind of "quantum stethoscope"). Moreover, the positive part of the spectrum of the initial Schrödinger operator is fully determined by the conductance properties of an "electronic device" consisting of the initial manifold and two "wires" attached to it (Propositions 8 and 9). We can choose the boundary conditions in such a way, that in the limiting case when wires are attached at the same point, the scattering matrix coincides with that for the $\delta^{\prime}$-interaction on the line. This fact is related to a conjecture from [5]: the scattering on the " $\delta^{\prime}$-potential" may be realized geometrically. Finally, in Sec. 5 we give a series of examples in which the Krein Q-function entering our expressions for the scattering matrix may be obtained in explicit form. Note that some applications of the results of this paper to the conductance of the quantum sphere were considered recently in [11]. Some aspects of the geometric scattering on non-compact Riemannian manifolds within the framework of the approach presented here are discussed in [10].

\section{Preliminaries}

Here we rephrase some results of operator extension theory using the language of boundary value spaces and linear symplectic geometry (see, e.g. [18], [35], 441, 442, 447, [60] for details). 
Let $V$ be a complex vector space with a skew-Hermitian sesquilinear form $[x \mid y]$. The orthogonality with respect to this form will be denoted by $[\perp]: x[\perp] y$ means that $[x \mid y]=0$; the orthogonal complement of a set $X \subset V$ is denoted as $X^{[\perp]}$. A subspace $\Lambda \subset V$ is called isotropic (respectively, Lagrangian) if $\Lambda \subset \Lambda^{[\perp]}$ (respectively, $\Lambda=\Lambda^{[\perp]}$ ). If $\mathcal{H}$ is a Hilbert

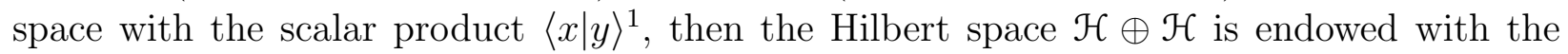
standard skew-Hermitian form

$$
[x \mid y]=\left\langle x_{1} \mid y_{2}\right\rangle-\left\langle x_{2} \mid y_{1}\right\rangle
$$

i.e. $[x \mid y]=\langle x \mid J y\rangle$, where $J: \mathcal{H} \oplus \mathcal{H} \rightarrow \mathcal{H} \oplus \mathcal{H}$ is a unitary operator of the form $J\left(x_{1}, x_{2}\right)=$ $\left(x_{2},-x_{1}\right)$. It is clear that $[x \mid y]$ is a continuous sesquilinear form on the Hilbert space $\mathcal{H} \oplus \mathcal{H}$, hence, every Lagrangian subspace in $\mathcal{H} \oplus \mathcal{H}$ is closed. Moreover, for every subset $X \subset \mathcal{H} \oplus \mathcal{H}$ we have $X^{[\perp]}=(J X)^{\perp}=J\left(X^{\perp}\right)$, where $X^{\perp}$ is the orthogonal complement with respect to the standard scalar product $\langle x \mid y\rangle$ in $\mathcal{H} \oplus \mathcal{H}:\langle x \mid y\rangle=\left\langle x_{1} \mid y_{1}\right\rangle+\left\langle x_{2} \mid y_{2}\right\rangle$. Therefore, a subspace $\Lambda \subset \mathcal{H} \oplus \mathcal{H}$ is isotropic (respectively, Lagrangian) iff $J \Lambda \subset \Lambda^{\perp}$ (respectively, $J \Lambda=\Lambda^{\perp}$ ).

For every skew-Hermitian sesquilinear form $[x \mid y]$ the form $i[x \mid y]$ is Hermitian; therefore the geometry of a skew-Hermitian sesquilinear form does not differ from that of a Hermitian form. Nevertheless, the symplectic language is very useful in operator extension theory. For example, let $A: \mathcal{D}(A) \rightarrow \mathcal{H}$ be a densely defined linear operator in $\mathcal{H}$ with the graph $\operatorname{Gr}(A), \operatorname{Gr}(A) \subset \mathcal{H} \oplus \mathcal{H}$. Then it is easy to check the following statements:

(1) $A$ is symmetric if and only if $\operatorname{Gr}(A)$ is an isotropic subspace of $\mathcal{H} \oplus \mathcal{H}$.

(2) $A$ is self-adjoint if and only if $\operatorname{Gr}(A)$ is a Lagrangian subspace of $\mathcal{H} \oplus \mathcal{H}$.

Remark 1. It is clear that every Lagrangian subspace is a maximal isotropic subspace, the converse is not true even in the one-dimensional case. On the other hand, if $V$ is a finite-dimensional complex space having at least one Lagrangian subspace, then according to the Witt theorem [7], every maximal isotropic subspace is Lagrangian. Therefore, in the finite-dimensional space $V=\mathcal{H} \oplus \mathcal{H}$ every maximal isotropic subspace is Lagrangian. On the contrary, let $\mathcal{H}$ be an infinite-dimensional Hilbert space, and let $A$ be a maximal symmetric operator in $\mathcal{H}$ which is not self-adjoint. Then $\operatorname{Gr}(A)$ is a maximal isotropic subspace of $\mathcal{H} \oplus \mathcal{H}$ which is not Lagrangian.

A linear mapping $u: V_{1} \rightarrow V_{2}$ of complex vector spaces $V_{1}, V_{2}$ with skew-Hermitian forms $[\cdot \mid \cdot]_{1},[\cdot \mid \cdot]_{2}$, respectively, is called skew-unitary if $[u(x) \mid u(y)]_{2}=[x \mid y]_{1} \forall x, y \in V_{1}$. Now let $S$ be a symmetric operator in $\mathcal{H}$; in the graph $\operatorname{Gr}\left(S^{*}\right)$ of $S^{*}$ we shall consider the skew-Hermitian form induced by the standard form from $\mathcal{H} \oplus \mathcal{H}$. A pair $(\mathcal{G}, \Gamma)$, where $\mathcal{G}$ is a Hilbert space and $\Gamma$ is a surjective skew-unitary mapping from $\operatorname{Gr}\left(S^{*}\right)$ onto $\mathcal{G} \oplus \mathcal{G}$ is called a boundary value space for $S$. It is known that a boundary value space for $S$ exists if and only if the deficiency indices $n_{+}(S)$ and $n_{-}(S)$ for $S$ coincide, i.e. if and only if $S$ has a self-adjoint extension. If this is the case and $(\mathcal{G}, \Gamma)$ is a boundary value space for $S$, then $\operatorname{dim} \mathcal{G}=n_{+}(S)\left(=n_{-}(S)\right)$ and $\Gamma$ is a continuous operator with respect to the standard Hilbert space topologies in $\operatorname{Gr}\left(S^{*}\right)$ and $\mathcal{G} \oplus \mathcal{G}$. Let $\widehat{\Gamma}: \mathcal{D}\left(S^{*}\right) \rightarrow \mathcal{G} \oplus \mathcal{G}$ be the composition of the canonical bijection $\mathcal{D}\left(S^{*}\right) \rightarrow \operatorname{Gr}\left(S^{*}\right)\left(x \mapsto\left(x, S^{*} x\right)\right)$ and $\Gamma$; it is clear that $\widehat{\Gamma}$ is surjective. Moreover, if $\mathcal{D}\left(S^{*}\right)$ is endowed with the graph scalar product $\langle x \mid y\rangle_{S}=\langle x \mid y\rangle+\left\langle S^{*} x \mid S^{*} y\right\rangle$,

\footnotetext{
${ }^{1}$ Throughout this paper, we assume that the scalar product is linear with respect to the second argument
} 
then $\widehat{\Gamma}$ is continuous. Denote by $P_{1}$ and $P_{2}$ the canonical projections of $\mathcal{G} \oplus \mathcal{G}$ onto $\mathcal{G} \oplus\{0\}$ and $\{0\} \oplus \mathcal{G}$, respectively, and by $\Gamma^{(1)}, \Gamma^{(2)}$ the operators $P_{1} \widehat{\Gamma}$ and $P_{2} \widehat{\Gamma}$, respectively. Then for all $x, y \in \mathcal{D}\left(S^{*}\right)$ the following relation takes place:

$$
\left\langle x \mid S^{*} y\right\rangle-\left\langle S^{*} x \mid y\right\rangle=\left\langle\Gamma^{(1)} x \mid \Gamma^{(2)} y\right\rangle-\left\langle\Gamma^{(2)} x \mid \Gamma^{(1)} y\right\rangle .
$$

Conversely, a triple $\left(\mathcal{G}, \Gamma^{(1)}, \Gamma^{(2)}\right)$, where $\Gamma$ is a Hilbert space and $\Gamma^{(j)}: \mathcal{D}\left(S^{*}\right) \rightarrow \mathcal{G}(j=1,2)$ are linear operators, uniquely defines a boundary value space, if the mapping $\mathcal{D}\left(S^{*}\right) \ni x \mapsto$ $\left(\Gamma^{(1)} x, \Gamma^{(2)} x\right) \in \mathcal{G} \oplus \mathcal{G}$ is surjective and the condition (1) holds. Indeed, it is sufficient to define $\Gamma$ by the rule $\Gamma\left(x, S^{*} x\right)=\left(\Gamma^{(1)} x, \Gamma^{(2)} x\right)$. The triple $\left(\mathcal{G}, \Gamma^{(1)}, \Gamma^{(2)}\right)$ is also called a boundary value space for $S$.

The following theorem describes all self-adjoint extensions of $S$ with help of the boundary value space.

Theorem A. Let $S$ be a symmetric operator in a Hilbert space $\mathcal{H}$ with coinciding deficiency indices, and let $(\mathcal{G}, \Gamma)$ be a boundary value space for $S$. Then for every Lagrangian subspace $\Lambda \subset \mathcal{G} \oplus \mathcal{G}$ the set $\Gamma^{-1}(\Lambda)$ is the graph of a self-adjoint operator $H^{\Lambda}$ that is a selfadjoint extension of $S$. Moreover, the correspondence $\Lambda \mapsto H^{\Lambda}$ is a bijection between all Lagrangian subspaces of $\mathcal{G} \oplus \mathcal{G}$ and all self-adjoint extensions of $S$.

In other words, the self-adjoint extension $H^{\Lambda}$ is defined by the boundary condition

$$
\left(\Gamma^{(1)} x, \Gamma^{(2)} x\right) \in \Lambda
$$

More precisely, the domain of $H^{\Lambda}$ is the subspace of $\mathcal{D}\left(S^{*}\right)$ given by $\mathcal{D}\left(H^{\Lambda}\right)=\left\{x \in \mathcal{D}\left(S^{*}\right)\right.$ : $\left.\left(\Gamma^{(1)} x, \Gamma^{(2)} x\right) \in \Lambda\right\}$, and $H^{\Lambda}$ is the restriction of $S^{*}$ to $\mathcal{D}\left(H^{\Lambda}\right)$. Condition (2) can be written in a more convenient "operator" form. Namely, for every Lagrangian subspace $\Lambda \subset \mathcal{G} \oplus \mathcal{G}$ there exists a uniquely defined unitary operator $U_{\Lambda}$ acting in $\mathcal{G}$ such that the relations $\left(x_{1}, x_{2}\right) \in \Lambda$ and $i\left(I+U_{\Lambda}\right) x_{1}=\left(I-U_{\Lambda}\right) x_{2}$ are equivalent; $U_{\Lambda}$ is called the Cayley transform for $\Lambda$. (If $\Lambda$ is the graph of a self-adjoint operator $L$ in $\mathcal{G}$, then $U_{\Lambda}$ is just the Cayley transform for $L)$. Moreover, the correspondence $\Lambda \mapsto U_{\Lambda}$ is a bijection between the sets of all Lagrangian subspaces of $\mathcal{G} \oplus \mathcal{G}$ and all unitary operators in $\mathcal{G}$. Using the notations above we can rewrite condition (2) in the desired operator form:

$$
\left(I-U_{\Lambda}\right) \Gamma^{(2)} x=i\left(I+U_{\Lambda}\right) \Gamma^{(1)} x .
$$

It is clear that a given Lagrangian subspace $\Lambda \subset \mathcal{G} \oplus \mathcal{G}$ has different equations of the form $L x_{1}=M x_{2}$, where $L$ and $M$ are bounded linear operators in $\mathcal{G}$. Hence, a given boundary condition $\left(\Gamma^{(1)} x, \Gamma^{(2)} x\right) \in \Lambda$ may be represented in the operator form

$$
L \Gamma^{(1)} x=M \Gamma^{(2)} x
$$

in many ways. Denote by $A(L, M)$ the bounded operator from $\mathcal{G} \oplus \mathcal{G}$ to $\mathcal{G}$ taking $x=$ $\left(x_{1}, x_{2}\right) \in \mathcal{G} \oplus \mathcal{G}$ to $L x_{1}-M x_{2} \in \mathcal{G}$.

Proposition B. Let $L, M: \mathcal{G} \rightarrow \mathcal{G}$ be bounded linear operators. The subspace $\Lambda$ of $\mathcal{G} \oplus \mathcal{G}$ determined by the equation $L x_{1}=M x_{2}$ is Lagrangian if and only if the following 
conditions are satisfied: (a) $L M^{*}=M L^{*}$; (b) the restriction of $A(L, M)$ to the subspace $J(\Lambda)$ is injective.

Proof. First of all we prove the equivalence of the following assertions:

(1) $\Lambda \supset \Lambda^{[\perp]}$; (2) $L M^{*}=M L^{*}$.

Indeed, by definition $\Lambda=\operatorname{Ker} A(L, M)$; on the other hand we have the wellknown relation $\operatorname{Ker} A(L, M)^{\perp}=\overline{\operatorname{Ran} A(L, M)^{*}}$. Since $\operatorname{Ker} A(L, M)$ is closed, condition (1) is equivalent to the condition (3) $J(\operatorname{Ker} A(L, M)) \supset \operatorname{Ran} A(L, M)^{*}$. Because $A(L, M)^{*} x=$ $\left(L^{*} x,-M^{*} x\right)$ for every $x \in \mathcal{G}$, the equivalence of (2) and (3) follows immediately.

Now let $\Lambda$ be a Lagrangian subspace, then $J(\Lambda)=\operatorname{Ker} A(L, M)^{\perp}$; therefore, the restriction of $A(L, M)$ to $J(\Lambda)$ is obviously injective. On the other hand, if conditions (a) and (b) are satisfied, then $J(\Lambda) \supset \Lambda^{\perp}$. Moreover, if $J(\Lambda) \neq \Lambda^{\perp}$, then $J(\Lambda)$ contains a non-zero element from $\operatorname{Ker} A(L, M)$, and we have a contradiction with (b).

Note that a finite-dimensional version of Proposition B has been given in 44, based on different arguments.

The self-adjoint extensions of $S$ defined by the conditions $\Gamma^{(1)} x=0$ and $\Gamma^{(2)} x=0$ will be denoted by $H^{(1)}$ and $H^{(2)}$, respectively; they correspond to the Lagrangian subspaces $\{0\} \oplus \mathcal{G}$ and $\mathcal{G} \oplus\{0\}$, respectively. If $\Lambda$ is the graph of a self-adjoint operator $L$ in $\mathcal{G}$ (i.e. if $\Lambda$ is transversal to $\{0\} \oplus \mathcal{G}: \Lambda \cap(\{0\} \oplus \mathcal{G})=\{0\})$, then the condition (2) takes the simpler form

$$
\Gamma^{(2)} x=L \Gamma^{(1)} x .
$$

The self-adjoint extension $H^{\Lambda}$ of $S$ is defined by a boundary condition of the form (四) with a self-adjoint $L$, if and only if $H^{\Lambda}$ is disjoint from $H^{(1)}$ (this means that $\mathcal{D}\left(H^{(1)}\right) \cap \mathcal{D}\left(H^{\Lambda}\right)=$ $\mathcal{D}(S))$.

On the other hand, at least in the case of a finite-dimensional $\mathcal{G}$ we can always define a given extension $H^{\Lambda}$ by a condition of the form (4). This may be done with the help of the above mentioned Witt theorem, but a more useful way is to use the complex version of the Arnold Lemma [3]. To state this lemma we need some auxiliary notations. Let $\mathbf{e}_{1}, \ldots, \mathbf{e}_{n}$ be a fixed orthonormal basis in $\mathcal{G}$, then the vectors $\mathbf{a}_{j}=\left(\mathbf{e}_{j}, 0\right)$ and $\mathbf{b}_{j}=\left(0, \mathbf{e}_{j}\right)(j=1, \ldots, n)$ form a symplectic basis in $\mathcal{G} \oplus \mathcal{G}$ :

$$
\left[\mathbf{a}_{j} \mid \mathbf{a}_{k}\right]=\left[\mathbf{b}_{j} \mid \mathbf{b}_{k}\right]=0 \quad\left[\mathbf{a}_{j} \mid \mathbf{b}_{k}\right]=-\left[\mathbf{b}_{k} \mid \mathbf{a}_{j}\right]=\delta_{j k}
$$

Let $\eta$ be a subset of $\{1, \ldots, n\}, \eta^{\prime}=\{1, \ldots, n\} \backslash \eta$; by virtue of (5) the linear hull of the set $\left\{\mathbf{a}_{j}: j \in \eta\right\} \cup\left\{\mathbf{b}_{j}: j \in \eta^{\prime}\right\}$, is a Lagrangian subspace of $\mathcal{G} \oplus \mathcal{G}$ which is called a coordinate subspace and denoted by $\mathcal{G}_{\eta}$. It is clear that if $\eta=\{1, \ldots, n\}$, then $\mathcal{G} \oplus\{0\}=\mathcal{G}_{\eta}$, $\{0\} \oplus \mathcal{G}=\mathcal{G}_{\eta^{\prime}}$.

Proposition C (Arnold's Lemma). Let $\mathcal{G}$ be finite-dimensional. Then every Lagrangian subspace of $\mathcal{G} \oplus \mathcal{G}$ is transversal to some coordinate subspace.

Moreover, $\mathcal{G} \oplus \mathcal{G}=\mathcal{G}_{\eta} \oplus \mathcal{G}_{\eta^{\prime}}$ where the sums are orthogonal with respect to the standard scalar product $\langle x \mid y\rangle$ in $\mathcal{G} \oplus \mathcal{G}$. Denote the orthoprojection of $\mathcal{G} \oplus \mathcal{G}$ onto $\mathcal{G}_{\eta}$ by $P_{\eta}$; by $J_{\eta}^{(1)}$ we shall denote the isomorphism of $\mathcal{G}_{\eta}$ onto $\mathcal{G}$ which takes the elements from $\mathcal{G}_{\eta}$ of the form $\mathbf{a}_{j}$ or $\mathbf{b}_{j}$ to $\mathbf{e}_{j}$, by $J_{\eta}^{(2)}$ we denote the isomorphism of $\mathcal{G}_{\eta^{\prime}}$ onto $\mathcal{G}$ which takes the 
elements from $\mathcal{G}_{\eta}$ of the form $\mathbf{a}_{j}$ to $-\mathbf{e}_{j}$ and of the form $\mathbf{b}_{j}$ into $\mathbf{e}_{j}$. Let now $(\mathcal{G}, \Gamma)$ be a boundary value space for a symmetric operator $S$, denote $\Gamma_{\eta}^{(1)}=J_{\eta}^{(1)} P_{\eta} \Gamma, \Gamma_{\eta}^{(2)}=J_{\eta}^{(2)} P_{\eta} \Gamma$. Then the triple $\left(\mathcal{G}, \Gamma_{\eta}^{(1)}, \Gamma_{\eta}^{(2)}\right)$ is a boundary value space for $S$ as well. For example, if $\eta=\{1, \ldots, n\}$, then $\Gamma_{\eta}^{(j)}=\Gamma^{(j)}$; on the other hand, $\Gamma_{\emptyset}^{(1)}=\Gamma^{(2)}, \Gamma_{\emptyset}^{(2)}=-\Gamma^{(1)}$.

By virtue of the Arnold lemma, for every Lagrangian subspace $\Lambda \subset \mathcal{G} \oplus \mathcal{G}$ there exists $\eta \subset\{1, \ldots, n\}$ such that the self-adjoint extension $H^{\Lambda}$ is given by the boundary condition of the form $\Gamma_{\eta}^{(2)} x=L \Gamma_{\eta}^{(1)} x$ where $L$ is a self-adjoint operator in $\mathcal{G}$. We shall denote this extension by $H^{L, \eta}$; the representation of $H^{\Lambda}$ in the form $H^{L, \eta}$ is, clearly, not unique. The extensions of $S$ defined by the conditions $\Gamma_{\eta}^{(j)} x=0(j=1,2)$ will be defined by $H_{\eta}^{(j)}$.

There exists a very convenient expression for the resolvent $R^{\Lambda}(z)=\left(H^{\Lambda}-z\right)^{-1}$ of the operator $H^{\Lambda}$ which is given by the so-called Krein resolvent formula. To give this formula, we need some preliminary notions (details may be found in [18], [51]). Let $z \in \mathbb{C} \backslash \mathbb{R}$, denote by $\mathcal{N}_{z}$ the deficiency subspace for $S: \mathcal{N}_{z}=\operatorname{Ker}\left(S^{*}-z\right)$. It may be proven that the restrictions of both the operators $\Gamma^{(j)}(j=1,2)$ to $\mathcal{N}_{z}$ are linear-topological isomorphisms of $\mathcal{N}_{z}$ onto G; we denote these restrictions as $\Gamma^{(j)}(z)$. Moreover, the operators $\gamma(z)=\left(\Gamma^{(1)}(z)\right)^{-1}$ form a holomorphic family of elements from the Banach space $\mathcal{L}(\mathcal{G}, \mathcal{H})$ of all linear continuous operators from $\mathcal{G}$ to $\mathcal{H}$. Further, the operators $Q(z)=\Gamma^{(2)} \gamma(z)$ form a holomorphic family in the Banach space $\mathcal{L}(\mathcal{G}, \mathcal{G})$. The holomorphic operator-valued functions $z \mapsto \gamma(z)$ and $z \mapsto Q(z)$ have analytic continuations on the set $\rho\left(H^{(1)}\right)$ of the regular values of $H^{(1)}$ : $\rho\left(H^{(1)}\right)=\mathbb{C} \backslash \sigma\left(H^{(1)}\right)$. This assertion follows from the relations below, which are valid for every $z, \zeta \in \mathbb{C} \backslash \mathbb{R}$ :

$$
\begin{gathered}
\gamma(z)=\gamma(\zeta)+(z-\zeta)\left(H^{(1)}-z\right)^{-1} \gamma(\zeta) ; \\
Q(z)-Q(\zeta)=(z-\zeta) \gamma^{*}(\bar{\zeta}) \gamma(z) .
\end{gathered}
$$

The functions $\gamma: \rho\left(H^{(1)}\right) \rightarrow \mathcal{L}(\mathcal{G}, \mathcal{H})$ and $Q: \rho\left(H^{(1)}\right) \rightarrow \mathcal{L}(\mathcal{G}, \mathcal{G})$ are called Krein $\Gamma$-field and Krein Q-function of the operator $S$ associated with the boundary value space $(\mathcal{G}, \Gamma)$.

Further we shall consider a subspace $\Lambda \subset \mathcal{G} \oplus \mathcal{G}$ as the graph of a multi-valued linear operator $M_{\Lambda}$ with the domain $\mathcal{D}\left(M_{\Lambda}\right)=P_{1}(\Lambda)$. The operator $M_{\Lambda}$ takes each $x \in \mathcal{D}\left(M_{\Lambda}\right)$ to an affine subspace $\{y \in \mathcal{G}:(x, y) \in \Lambda\}$ of $\mathcal{G}$. For every subspace $\Lambda \subset \mathcal{G} \oplus \mathcal{G}$ we denote by $\Lambda^{-1}$ the "inverse" subspace $\Lambda^{-1}=\{(x, y) \in \mathcal{G} \oplus \mathcal{G}:(y, x) \in \Lambda\}$. In particular, if $\Lambda$ is the graph of an invertible operator $L: \mathcal{D}(L) \rightarrow \mathcal{G}$, then $\Lambda^{-1}$ is the graph of the inverse operator $L^{-1}$. In the following we shall identify mappings and their graphs if this does not lead to ambiguities.

Theorem D. Let $S$ be a symmetric operator in a Hilbert space $\mathcal{H}$ with boundary value space $(\mathcal{G}, \Gamma)$, and let $\gamma$ and $Q$ be the corresponding $\Gamma$-field and Q-function for $S$, respectively. Suppose that $H^{\Lambda}$ is a self-adjoint extension of $S$ associated with a Lagrangian subspace $\Lambda$ of $\mathcal{G} \oplus \mathcal{G}$. Then for every $z \in \rho\left(H^{(1)}\right) \cap \rho\left(H^{\Lambda}\right)$ the subspace $[Q(z)-\Lambda]^{-1}$ is the graph of a bounded (single-valued) operator in $\mathcal{G}$ and the resolvent of $R^{\Lambda}(z)=\left(H^{\Lambda}-z\right)^{-1}$ has the form

$$
R^{\Lambda}(z)=R^{(1)}(z)-\gamma(z)[Q(z)-\Lambda]^{-1} \gamma^{*}(\bar{z})
$$


where $R^{(1)}(z)=\left(H^{(1)}-z\right)^{-1}$ is the resolvent of $H^{(1)}$.

If $H^{\Lambda}$ and $H^{(1)}$ are not disjoint, then the Krein formula (6) contains a multi-valued operator $\Lambda$. To avoid the use of such operators we can proceed as follows [51]. Let $\Lambda$ be a Lagrangian subspace of $\mathcal{G} \oplus \mathcal{G}$ and $U_{\Lambda}$ be its Cayley transform. Denote by $P_{\Lambda}$ the orthogonal projection of $\mathcal{G}$ onto subspace $\mathcal{G}_{\Lambda}=\overline{\operatorname{Ran}\left(U_{\Lambda}-I\right)}$, by $J_{\Lambda}$ the canonical embedding of $\mathcal{G}_{\Lambda}$ into $\mathcal{G}$, and by $I_{\Lambda}$ the identity operator in $\mathcal{G}_{\Lambda}$. Then $V_{\Lambda}=P_{\Lambda} U_{\Lambda} J_{\Lambda}$ is a unitary operator in $\mathcal{G}_{\Lambda}$, and 1 is not an eigenvalue of this operator. Therefore, $L=i\left(I_{\Lambda}+V_{\Lambda}\right)\left(I_{\Lambda}-V_{\Lambda}\right)^{-1}$ is a self-adjoint operator in $\mathcal{G}_{\Lambda}$, and

$$
[Q(z)-\Lambda]^{-1}=J_{\Lambda}\left[P_{\Lambda} Q(z) J_{\Lambda}-L\right]^{-1} P_{\Lambda} .
$$

Moreover, the following proposition holds [51]:

Proposition E. Let $L_{n}$ be a self-adjoint operator in $\mathcal{G}$ of the form $L_{n}=J_{\Lambda} L P_{\Lambda}+$ $n\left(I-P_{\Lambda}\right)$. Then for every $z \in \rho\left(H^{(1)}\right) \cap \rho\left(H^{\Lambda}\right)$

$$
\lim _{n}\left[Q(z)-L_{n}\right]^{-1}=J_{\Lambda}\left[P_{\Lambda} Q(z) J_{\Lambda}-L\right]^{-1} P_{\Lambda}
$$

in the strong operator topology.

If $\mathcal{G}$ is finite-dimensional, then we can adapt the Arnold Lemma to avoid the use of multi-valued mappings in the Krein formula. Namely, denote the Krein $\Gamma$-field and Qfunction for the boundary value space $\left(\mathcal{G}, \Gamma_{\eta}^{(1)}, \Gamma_{\eta}^{(2)}\right)$ by $\gamma_{\eta}(z)$ and $Q_{\eta}(z)$, respectively. Since $H^{\Lambda}$ coincides with some operator of the form $H^{L, \eta}$, then (6) may be rewritten in the form

$$
R^{\Lambda}(z) \equiv R^{L, \eta}(z)=R_{\eta}^{(1)}(z)-\gamma_{\eta}(z)\left[Q_{\eta}(z)-L\right]^{-1} \gamma_{\eta}^{*}(\bar{z}),
$$

where $R_{\eta}^{(1)}(z)=\left(H_{\eta}^{(1)}-z\right)^{-1}$.

\section{Schrödinger operator on a "hedgehog shaped" space}

Consider a complete (not necessarily connected) Riemannian manifold $X$ of dimension $d$, with metric $g_{\mu \nu}$. We shall denote by $g$ the determinant $\operatorname{det}\left(g_{\mu \nu}\right)$, by $d \lambda$ the Riemannian measure, and by $r(x, y)$ the geodesic distance on $X$. Fix a non-empty finite subset $\left\{q_{1}, \ldots, q_{n}\right\}$ of $X$, and let $\mathbb{R}_{+}^{(j)}(j=1, \ldots, n)$ be copies of the half-line $\mathbb{R}_{+}=\{x \in \mathbb{R}: x \geq 0\}$. Let $\widehat{X}$ be the topological space obtained from the disjoint union $X \sqcup \mathbb{R}_{+}^{(1)} \sqcup \ldots \sqcup \mathbb{R}_{+}^{(n)}$ by gluing the point $0 \in \mathbb{R}_{+}^{(j)}$ to the point $q_{j}$. The "hedgehog shaped" topological space $\widehat{X}$ may be considered as a limit of manifolds with $n$ horns as the widths of the horns tend to zero. Let $\mathcal{H}_{0}:=L^{2}(X, d \lambda), \mathcal{H}_{j}:=L^{2}\left(\mathbb{R}_{+}^{(j)}, d x\right)$. The sum of the Riemannian measure $d \lambda$ on $X$ and the Lebesgue measures $d x$ on $\mathbb{R}_{+}^{(j)}$ is a natural measure $d \mu$ on $\widehat{X}$; the space $L^{2}(\widehat{X}, d \mu)$ will be identified with the space $\mathcal{H}:=\mathcal{H}_{0} \oplus \mathcal{H}_{1} \oplus \ldots \oplus \mathcal{H}_{n}$.

To define a Schrödinger operator on $\widehat{X}$ we proceed as follows. Consider the symmetric operator $\tau$ in $\mathcal{H}_{0}$, with domain $C_{0}^{\infty}(X)$, defined by the differential expression

$$
\tau=-g^{-1 / 2}(x)\left(\partial_{\mu}+i \mathcal{A}_{\mu}(x)\right) g^{1 / 2}(x) g^{\mu \nu}(x)\left(\partial_{\nu}+i \mathcal{A}_{\nu}(x)\right)+p(x),
$$


where $\mathcal{A}_{\mu}(\mu=1, \ldots, d)$ and $p$ are real-valued smooth functions on $X\left(\mathcal{A}_{\mu}\right.$ are the components of a vector potential $\mathcal{A}$ of a certain magnetic field on $X$, and $p$ is the scalar potential of an electric field). We denote the closure of $\tau$ in $\mathcal{H}_{0}$ by $H_{0}$, and suppose that the potentials $\mathcal{A}$ and $p$ are chosen in such a way that $H_{0}$ is a self-adjoint operator in $\mathcal{H}_{0}$, i.e. we assume that $\tau$ is essentially self-adjoint. Note that this is the case, if $\mathcal{A}_{\mu}$ and $p$ have compact supports, in particular, if $X$ is compact. If $\mathcal{A}=0$ and $p=0$ we get the Laplace-Beltrami operator $-\Delta_{X}$ on $X$. To use the techniques of the operator extension theory we need the condition

(C) $\mathcal{D}\left(H_{0}\right)$ imbeds in $C(X)$.

By virtue of the well-known Sobolev embedding theorems, this condition is satisfied if and only if $d \leq 3$. Therefore, from this point on we suppose that $0<d \leq 3$.

Let now $S_{0}$ be the operator in $\mathcal{H}_{0}$ that is the restriction of $H_{0}$ to the domain

$$
\mathcal{D}\left(S_{0}\right)=\left\{f \in \mathcal{D}\left(H_{0}\right): f\left(q_{j}\right)=0 \quad \forall j=1, \ldots, n\right\} .
$$

It is clear that $S_{0}$ is symmetric in $\mathcal{H}_{0}$, and it is easy to prove that the deficiency indices of $S_{0}$ are $(n, n)$. Denote next by $S_{j}(j=1, \ldots, n)$ the closure in $\mathcal{H}_{j}$ of the operator $-d^{2} / d x^{2}$ defined on $C_{0}^{\infty}(0, \infty) ; S_{j}$ is a symmetric operator in $\mathcal{H}_{j}$ with the deficiency indices $(1,1)$. Finally, we set $S:=S_{0} \oplus S_{1} \oplus \ldots \oplus S_{n}$; it is evident that $S$ is a symmetric operator in $\mathcal{H}$ with deficiency indices $(2 n, 2 n)$.

Definition. Any self-adjoint extension $H$ of the operator $S$ we shall call a Schrödinger operator on $\widehat{X}$ with vector potential $\mathcal{A}$ and scalar potential $p$.

According to the theory presented in Sec. 1, to describe all the Schrödinger operators on $\widehat{X}$ with given vector and scalar potentials we must construct a boundary value space for $S$. For this purpose we construct boundary value spaces for the operators $S_{0}, S_{1}, \ldots ., S_{n}$ and take the direct sum of these spaces. Let us start with a simple case of the operators $S_{j}$ $(j=1, \ldots, n)$.

Lemma 1. Set $\mathcal{G}_{j}=\mathbb{C}$ and define the operators $\Gamma_{j}^{(1)}, \Gamma_{j}^{(2)} \in \mathcal{L}\left(\mathcal{D}\left(S_{j}^{*}\right), \mathcal{G}_{j}\right), j=$ $1, \ldots, n$, by the rule:

$$
\Gamma_{j}^{(1)}(f)=-f^{\prime}(0), \quad \Gamma_{j}^{(2)}(f)=f(0) .
$$

Then the triple $\left(\mathcal{G}_{j}, \Gamma_{j}^{(1)}, \Gamma_{j}^{(2)}\right)$ is a boundary value space for $S_{j}$.

We omit the simple proof.

It is clear that $H_{j}^{\mathrm{N}}:=H_{j}^{(1)}$ and $H_{j}^{\mathrm{D}}:=H_{j}^{(2)}$ is the free Schrödinger operator on the semi-axis $\mathbb{R}_{+}^{(j)}$ with the Neumann and Dirichlet boundary condition at the point $x=0$, respectively. Since the space $\mathcal{G}_{j}$ is one-dimensional, the corresponding $\Gamma$-field $\gamma_{j}(z)$ may be considered as a holomorphic function on $\mathbb{C} \backslash \mathbb{R}_{+}=\rho\left(H_{j}^{(1)}\right)$ with values in $\mathcal{H}_{j}=L^{2}\left(\mathbb{R}_{+}\right)$, and the corresponding Q-function $Q_{j}(z)$ as a holomorphic function in $\mathbb{C} \backslash \mathbb{R}_{+}$. It is clear that $\gamma_{j}$ and $Q_{j}$ are independent of $j$.

Lemma 2. The $\Gamma$-field and the Q-function for $S_{j}$ associated with the boundary value space $\left(\mathcal{G}_{j}, \Gamma_{j}^{(1)}, \Gamma_{j}^{(2)}\right)$ are given by:

$$
\gamma_{j}(z)(x)=\frac{1}{\sqrt{-z}} \exp (-\sqrt{-z} x)
$$




$$
Q_{j}(z)=\frac{1}{\sqrt{-z}} .
$$

Remark 2. Throughout the paper, the continuous branch of the square root is chosen in $\mathbb{C} \backslash(-\infty, 0)$, such that $\operatorname{Re} \sqrt{z}>0$ if $z \neq 0$.

Proof. It is clear that $S_{j}$ is the restriction of $H_{j}^{\mathrm{N}}$ to the domain $\left\{f \in \mathcal{D}\left(H_{j}^{\mathrm{N}}\right): f(0)=0\right\}$. On the other hand, it is easy to check that the Green function $G_{j}^{\mathrm{N}}(x, y ; z)$ of the Neumann operator $H_{j}^{\mathrm{N}}$ is given as

$$
G_{j}^{\mathrm{N}}(x, y ; z)=\frac{1}{2 \sqrt{-z}}[\exp (-\sqrt{-z}|x-y|)+\exp (-\sqrt{-z}(x+y))] .
$$

Hence, the function $g(x)=G_{j}^{\mathrm{N}}(x, 0 ; z)$ is a non-zero element of $\operatorname{Ker}\left(S_{j}^{*}-z\right)$. Since $-g^{\prime}(0)=1$, the operator $\Gamma_{j}^{(1)} \gamma_{j}(z)$ is the identity on $\mathcal{G}_{j}$. Therefore, $\gamma_{j}(z)$ is the $\Gamma$-field. The equation $Q_{j}(z)=\Gamma_{j}^{(2)} \gamma(z)$ is trivial, so $Q_{j}(z)$ is the Q-function.

Sometimes it is more convenient to use the boundary value space $\left(\mathcal{G}_{j}, \Gamma_{\emptyset, j}^{(1)}, \Gamma_{\emptyset, j}^{(2)}\right)$ (see notations in the preceding section). It is clear that $\Gamma_{\emptyset, j}^{(1)} f=f(0)$, and $\Gamma_{\emptyset, j}^{(2)} f=f^{\prime}(0)$; thus $H_{\emptyset, j}^{(1)}=H_{j}^{\mathrm{D}}, H_{\emptyset, j}^{(2)}=H_{j}^{\mathrm{N}}$. Using the definitions and Lemma 2, we get

Lemma 3. The $\Gamma$-field $\gamma_{j}^{\mathrm{D}}$ and $\mathcal{Q}$-function $Q_{j}^{\mathrm{D}}$ for $S_{j}$ associated with the boundary value space $\left(\mathcal{G}_{j}, \Gamma_{\emptyset, j}^{(1)}, \Gamma_{\emptyset, j}^{(2)}\right)$ have the following form:

$$
\begin{gathered}
\gamma_{j}^{\mathrm{D}}(z)(x) \equiv \gamma_{\emptyset, j}(z)(x)=\exp (-\sqrt{-z} x), \\
Q_{j}^{\mathrm{D}}(z) \equiv Q_{\emptyset, j}(z)=-\sqrt{-z} .
\end{gathered}
$$

Now we turn to the operator $S_{0}$. First of all, denote by $R_{0}(z)$ the resolvent for $H_{0}$, $R_{0}(z)=\left(H_{0}-z\right)^{-1}$; by $G_{0}(x, y ; z)$ we shall denote the Green function for $H_{0}$ (the integral kernel of $R_{0}(z)$ in the space $\left.L^{2}(X, d \lambda)\right)$. Fix $q \in X$ and $z \in \rho\left(H_{0}\right)$, then near $q$ the function $G_{0}(x, q ; z)$ has the expansion [4], [14], [57]:

$$
G_{0}(x, q ; z)=F_{0}(x, q)+F_{1}(x, q ; z)+R(x, q ; z),
$$

where $F_{0}$ is independent of the spectral parameter $z$ and has the following form:

$$
F_{0}(x, q)= \begin{cases}-\frac{c_{1}(x, q)}{2} r(x, q), & \text { if } d=1 ; \\ -\frac{c_{2}(x, q)}{2 \pi} \ln r(x, q), & \text { if } d=2 \\ \frac{c_{3}(x, q)}{4 \pi}[r(x, y)]^{-1}, & \text { if } d=3 .\end{cases}
$$

Here $c_{j}(x, q)(j=1,2,3)$ does not depend on $z$, is a continuous functions of $x$, and $c_{j}(q, q)=$ 1 ; moreover, $c_{1}$ is a smooth function of $x$. Further, the function $F_{1}$ is continuous with respect to $x$; as for the remainder term $R$, it has the following behavior near $q$ as $x \rightarrow q$ :

$$
R(x, q ; z)= \begin{cases}o(r(x, q)), & \text { if } d=1 \\ o(1), & \text { if } d=2 \text { or } d=3 .\end{cases}
$$


Finally, $F_{1}$ and $R$ are analytic functions of $z$ in the domain $\rho\left(H_{0}\right)=\mathbb{C} \backslash \sigma\left(H_{0}\right)$.

For $z \in \rho\left(H_{0}\right)$ define a matrix $Q_{0}(z)$ by the relations:

$$
\left[Q_{0}(z)\right]_{l m}:= \begin{cases}G_{0}\left(q_{l}, q_{m} ; z\right), & \text { if } l \neq m ; \\ F_{1}\left(q_{l}, q_{l} ; z\right), & \text { if } l=m\end{cases}
$$

(note that $\left[Q_{0}(z)\right]_{l m}=G_{0}\left(q_{l}, q_{m} ; z\right)$ for all $l$ and $m$, if $d=1$ ). Clearly, $Q_{0}(z)$ is a holomorphic matrix-valued function in the domain $\rho\left(H_{0}\right)$ obeying the condition

$$
{\overline{\left[Q_{0}(z)\right]_{l m}}}_{\left.l Q_{0}(\bar{z})\right]_{m l}} \text {. }
$$

The following assertion is needed below (see [48], 69], 30]).

Lemma 4. If $z \in \mathbb{C} \backslash \mathbb{R}$, then the functions $G_{0}\left(\cdot, q_{j} ; z\right), j=1, \ldots, n$, form a vector basis in the deficiency subspace $\mathcal{N}_{z}=\operatorname{Ker}\left(S_{0}^{*}-z\right)$.

Fix $z \in \mathbb{C} \backslash \mathbb{R}$, then $\mathcal{D}\left(S_{0}^{*}\right)=\mathcal{D}\left(\bar{S}_{0}\right) \dot{+} \mathcal{N}_{z} \dot{+} \mathcal{N}_{\bar{z}}$ (there is an algebraic direct sum here; if $z=i$, then this sum is orthogonal with respect to the scalar product $\left.\langle x \mid y\rangle_{S}\right)$. By Lemma 4 each function $f$ from $\mathcal{D}\left(S_{0}^{*}\right)$ has the following asymptotic expansion near the point $q_{j}$ :

$$
f(x)=a_{j}(f) F_{0}\left(x, q_{j}\right)+b_{j}(f)+R(x),
$$

where $a_{j}(f), b_{j}(f) \in \mathbb{C}$, and the behavior of the remainder term $R(x)$ is given by (15) as $x \rightarrow q_{j}$.

Lemma 5. Set $\mathcal{G}_{0}=\mathbb{C}^{n}$ and define operators $\Gamma_{0}^{(1)}, \Gamma_{0}^{(2)} \in \mathcal{L}\left(\mathcal{D}\left(S_{0}^{*}\right), \mathcal{G}_{0}\right)$ by

$$
\begin{aligned}
& \Gamma_{0}^{(1)}(f):=\left(a_{j}(f)\right)_{1 \leq j \leq n}, \\
& \Gamma_{0}^{(2)}(f):=\left(b_{j}(f)\right)_{1 \leq j \leq n},
\end{aligned}
$$

where $a_{j}(f)$ and $b_{j}(f)$ are the constants from (18). Then the triple $\left(\mathcal{G}_{0}, \Gamma_{0}^{(1)}, \Gamma_{0}^{(2)}\right)$ is a space of boundary values for $S_{0}$.

Proof. Since $C_{0}^{\infty}(X) \subset \mathcal{D}\left(H_{0}\right)$ it is easy to conclude that the mapping $f \mapsto\left(\Gamma_{0}^{(1)} f, \Gamma_{0}^{(2)} f\right)$ is surjective. It remains to prove the condition (11).

Consider the sesquilinear forms $B_{1}, B_{2}$ defined as follows:

$$
\begin{gathered}
B_{1}(f, g):=\left\langle f \mid S_{0}^{*} g\right\rangle-\left\langle S_{0}^{*} f \mid g\right\rangle \\
B_{2}(f, g):=\left\langle\Gamma_{0}^{(1)} f \mid \Gamma_{0}^{(2)} g\right\rangle-\left\langle\Gamma_{0}^{(2)} f \mid \Gamma_{0}^{(1)} g\right\rangle=\sum_{j=1}^{n}\left[\overline{a_{j}(f)} b_{j}(g)-\overline{b_{j}(f)} a_{j}(g)\right]
\end{gathered}
$$

and set

$$
g_{j}^{ \pm}(x)=G_{0}\left(x, q_{j} ; \pm i\right), \quad j=1, \ldots, n .
$$

It is easy to check the following properties of the functions $g_{j}^{ \pm}$:
(i) $S_{0}^{*} g_{j}^{ \pm}= \pm i g_{j}^{ \pm}$;
(ii) $a_{j}\left(g_{k}^{ \pm}\right)=\delta_{j k}$;
(iii) $\quad b_{j}\left(g_{k}^{ \pm}\right)=Q_{0}^{j k}( \pm i)$. 
To prove the lemma, it is enough to verify that $B_{1}(f, g)=B_{2}(f, g)$ for $f, g \in \mathcal{D}\left(S_{0}^{*}\right)$. Since $\mathcal{D}\left(S_{0}^{*}\right)=\mathcal{D}\left(\bar{S}_{0}\right) \oplus \mathcal{N}_{i} \oplus \mathcal{N}_{-i}$, it is enough to check the equality $B_{1}(f, g)=B_{2}(f, g)$ for all functions $f, g \in \mathcal{D}\left(S_{0}\right) \cup\left\{g_{j}^{ \pm}: j=1, \ldots, n\right\}$. It is clear that $a_{j}(f)=b_{j}(f)=0$ if $f \in \mathcal{D}\left(S_{0}\right)$; therefore $B_{1}(f, g)=0=B_{2}(f, g)$ if $f \in \mathcal{D}\left(S_{0}\right)$ or $g \in \mathcal{D}\left(S_{0}\right)$. By (i) from (19), $B_{1}\left(g_{j}^{+}, g_{k}^{-}\right)=0$ $\forall j, k \in\{1, \ldots, n\}$. On the other hand, Eqs. (ii) and (iii) from (19) and (17) imply that

$$
B_{2}\left(g_{j}^{+}, g_{k}^{-}\right)=b_{j}\left(g_{k}^{-}\right)-\overline{b_{k}\left(g_{j}^{+}\right)}=\left[Q_{0}(-i)\right]_{j k}-{\overline{\left[Q_{0}(i)\right.}}_{k j}=0 .
$$

Hence, $B_{1}\left(g_{j}^{+}, g_{k}^{-}\right)=B_{2}\left(g_{j}^{+}, g_{k}^{-}\right) \forall j, k \in\{1, \ldots, n\}$. Since $B_{l}(f, g)=-\overline{B_{l}(g, f)}(l=1,2)$, we have: $B_{1}\left(g_{j}^{-}, g_{k}^{+}\right)=B_{2}\left(g_{j}^{-}, g_{k}^{+}\right) \forall j, k \in\{1, \ldots, n\}$. Similarly, we get

$$
B_{2}\left(g_{j}^{+}, g_{k}^{+}\right)=b_{j}\left(g_{k}^{+}\right)-\overline{b_{k}\left(g_{j}^{+}\right)}=[Q(i)]_{j k}-\overline{[Q(i)}_{k j}=[Q(i)]_{j k}-[Q(-i)]_{j k} .
$$

Further

$$
\begin{gathered}
B_{1}\left(g_{j}^{+}, g_{k}^{+}\right)=2 i\left\langle g_{j}^{+} \mid g_{k}^{+}\right\rangle= \\
2 i \int_{X} \overline{G_{0}\left(x, q_{j} ; i\right)} G_{0}\left(x, q_{k} ; i\right) d \lambda(x)=2 i \int_{X} G_{0}\left(q_{j}, x ;-i\right) G_{0}\left(x, q_{k} ; i\right) d \lambda(x) .
\end{gathered}
$$

Using the Hilbert resolvent identity we obtain from (21) in case $j \neq k$ :

$$
B_{1}\left(g_{j}^{+}, g_{k}^{+}\right)=G_{0}\left(q_{j}, q_{k} ; i\right)-G_{0}\left(q_{j}, q_{k} ;-i\right)=B_{2}\left(g_{j}^{+}, g_{k}^{+}\right)
$$

If $j=k$, then using the Hilbert identity again we get

$$
\begin{gathered}
B_{1}\left(g_{j}^{+}, g_{j}^{+}\right)=2 i \lim _{q \rightarrow q_{j}} \int_{X} G_{0}(q, x ;-i) G_{0}\left(x, q_{j} ; i\right) d \lambda(x)= \\
\lim _{q \rightarrow q_{j}}\left[G_{0}\left(q, q_{j} ; i\right)-G_{0}\left(q, q_{j} ;-i\right)\right]= \\
{[Q(i)]_{j j}-[Q(-i)]_{j j}=B_{2}\left(g_{j}^{+}, g_{j}^{+}\right)}
\end{gathered}
$$

(of course, in the case $d=1$ we can omit the limiting procedure). The proof of the equalities $B_{1}\left(g_{j}^{-}, g_{k}^{-}\right)=B_{2}\left(g_{j}^{-}, g_{k}^{-}\right)$is similar.

Remark 3. It is clear that in the case $d=1$ we have $b_{j}(f)=f\left(q_{j}\right)$. Moreover, we can get a simple expression for $a_{j}(f)$ in this case. Namely, choose a chart $U \subset X$ such that $q_{j} \in U \forall$ $j=1, \ldots, n$ and $U$ is isometric to an interval $(a, b) \subset \mathbb{R}$. Using the Cartesian coordinates in $U$ we obtain from (18) that every function $f \in \mathcal{D}\left(S_{0}^{*}\right)$ has the following expansion near each point $q_{j}$ :

$$
f(x)=-\frac{1}{2} a_{j}(f)\left|x-q_{j}\right|+f\left(q_{j}\right)+o\left(\left|x-q_{j}\right|\right),
$$

thus it follows from (22) that

$$
a_{j}(f)=f^{\prime}\left(q_{j}-0\right)-f^{\prime}\left(q_{j}+0\right),
$$

where the derivative is taken with respect to the Cartesian coordinate in $U$. 
Now we describe the Krein $\Gamma$-field and Q-function for $S_{0}$ associated with the boundary value space $\left(\mathcal{G}_{0}, \Gamma_{0}^{(1)}, \Gamma_{0}^{(2)}\right)$.

Lemma 6. The Krein $\Gamma$-field for $S_{0}$ associated with the boundary value space $\left(\mathcal{G}_{0}, \Gamma_{0}^{(1)}, \Gamma_{0}^{(2)}\right)$ is an operator valued family $\gamma_{0}(z) \in \mathcal{L}\left(\mathcal{G}_{0}, \mathcal{H}_{0}\right)$ defined for an element $\zeta=$ $\left(\zeta_{j}\right)_{1 \leq j \leq n}$ from $\mathcal{G}_{0}=\mathbb{C}^{n}$ by

$$
\gamma_{0}(z)(\zeta)=\sum_{j=1}^{n} \zeta_{j} G_{0}\left(\cdot, q_{j} ; z\right) .
$$

The corresponding $\mathbf{Q}$-function coincides with the matrix-valued function $Q_{0}(z)$.

Proof. To prove the first part of the lemma, it is enough to check that $\Gamma_{0}^{(1)} \gamma_{0}(z)$ is the identity operator on $\mathcal{G}_{0}$, but this follows immediately from the definition of $\Gamma_{0}^{(1)}$ and from (13) and (14).

have

Let $g_{k}(x)=G_{0}\left(x, q_{k} ; z\right)$, then $b_{j}\left(g_{k}\right)=\left[Q_{0}(z)\right]_{j k}$ by definition. Thus for $\zeta \in \mathcal{G}_{0}$ we

$$
\left[\Gamma_{0}^{(2)} \gamma_{0}(z) \zeta\right]_{j}=\sum_{k=1}^{n}\left[Q_{0}(z)\right]_{j k} \zeta_{k}
$$

therefore, $Q_{0}(z)$ is the Q-function.

Now we set

$$
\begin{aligned}
\mathcal{G} & :=\mathcal{G}_{0} \oplus \mathcal{G}_{1} \oplus \ldots \oplus \mathcal{G}_{n}\left(=\mathbb{C}^{2 n}\right) \\
\Gamma^{(j)} & :=\Gamma_{0}^{(j)} \oplus \Gamma_{1}^{(j)} \oplus \ldots \oplus \Gamma_{n}^{(j)} \quad(j=1,2) ; \\
\gamma(z) & :=\gamma_{0}(z) \oplus \gamma_{1}(z) \oplus \ldots \oplus \gamma_{n}(z) \quad(z \in \mathbb{C} \backslash \mathbb{R}) \\
Q(z) & :=Q_{0}(z) \oplus Q_{1}(z) \oplus \ldots \oplus Q_{n}(z) \quad(z \in \mathbb{C} \backslash \mathbb{R}) .
\end{aligned}
$$

Then the following theorem is an evident consequence of the preceding lemmas.

Theorem 1. The triple $\left(\mathcal{G}, \Gamma^{(1)}, \Gamma^{(2)}\right)$ is a boundary value space for the operator $S$. The corresponding Krein $\Gamma$-field and Q-function coincide with $\gamma(z)$ and $Q(z)$, respectively. The operator $H=H^{(1)}$ given by the boundary condition $\Gamma^{(1)} f=0$ coincides with the direct sum $H=H_{0} \oplus H_{1}^{\mathrm{N}} \oplus \ldots \oplus H_{n}^{\mathrm{N}}$ (we shall denote this operator by $H_{\mathrm{N}}$ ).

Remark 4. It is convenient to describe explicitly the boundary value space $\left(\mathcal{G}, \Gamma_{\eta}^{(1)}, \Gamma_{\eta}^{(2)}\right)$ for an arbitrary set $\eta \subset\{1, \ldots, 2 n\}$. Denote

$$
\begin{aligned}
& \theta=\eta \cap\{1, \ldots, n\}, \quad \omega=\eta \cap\{n+1, \ldots, 2 n\}, \\
& \theta^{\prime}=\{1, \ldots, n\} \backslash \eta, \quad \omega^{\prime}=\{n+1, \ldots, 2 n\} \backslash \eta .
\end{aligned}
$$

Then

$$
\begin{aligned}
\Gamma_{\eta}^{(l)} & =\Gamma_{\theta, 0}^{(l)} \oplus \widetilde{\Gamma}_{1}^{(l)} \oplus \ldots \oplus \widetilde{\Gamma}_{n}^{(l)} \quad(l=1,2) \\
\gamma_{\eta}(z) & =\gamma_{\theta, 0}(z) \oplus \widetilde{\gamma}_{1}(z) \oplus \ldots \oplus \widetilde{\gamma}_{n}(z) \quad(z \in \mathbb{C} \backslash \mathbb{R}) \\
Q_{\eta}(z) & =Q_{\theta, 0}(z) \oplus \widetilde{Q}_{1}(z) \oplus \ldots \oplus \widetilde{Q}_{n}(z) \quad(z \in \mathbb{C} \backslash \mathbb{R}) .
\end{aligned}
$$


Here for $j=1, \ldots, n$

$$
\widetilde{\Gamma}_{j}^{(l)}= \begin{cases}\Gamma_{j}^{(l)}, & \text { if } j+n \in \omega, \\ \Gamma_{\emptyset, j}^{(l)}, & \text { if } j+n \in \omega^{\prime} ;\end{cases}
$$

and similarly for $\widetilde{\gamma}_{\eta}(z)$ and $\widetilde{Q}_{\eta}(z)$. In particular, if $\eta=\{1, \ldots, n\}$, then we denote $\Gamma_{\eta}^{(l)}=\Gamma_{\mathrm{D}}^{(l)}$, $\gamma_{\eta}=\gamma_{\mathrm{D}}, Q_{\eta}=Q_{\mathrm{D}}$. The operator $H=H_{\mathrm{D}}^{(1)}$ given by the boundary condition $\Gamma_{\mathrm{D}}^{(1)} f=0$ coincides with the direct sum $H=H_{0} \oplus H_{1}^{\mathrm{D}} \oplus \ldots \oplus H_{n}^{\mathrm{D}}$ and will be denoted by $H_{\mathrm{D}}$.

Now we can describe all Schrödinger operators on $\widehat{X}$ with given vector and scalar potentials in terms of boundary conditions at the points $q_{1}, \ldots, q_{n}$. First of all, we describe the elements of $\mathcal{D}\left(S^{*}\right)$ as functions on $\widehat{X}$. For $f \in L^{2}(\widehat{X})=\mathcal{H}$ we denote by $f_{0}, f_{1}, \ldots, f_{n}$ the components of $f$ in $L^{2}(X)=\mathcal{H}_{0}, L^{2}\left(\mathbb{R}_{+}^{(1)}\right)=\mathcal{H}_{1}, \ldots, L^{2}\left(\mathbb{R}_{+}^{(n)}\right)=\mathcal{H}_{n}$, respectively. It is clear that $f \in \mathcal{D}\left(S^{*}\right)$ if and only if $f_{j} \in H^{2}\left(\mathbb{R}_{+}\right)(j=1, \ldots, n)$ whereas $f_{0} \in H_{\mathrm{loc}}^{2}\left(X \backslash\left\{q_{1}, \ldots, q_{n}\right\}\right)$ and has the asymptotics (18) near each point $q_{j}$.

Theorem 2. The Schrödinger operators on $\widehat{X}$ with a given vector potential $\mathcal{A}$ and a given scalar potential $p$ are in bijective correspondence with the Lagrangian subspaces of $\mathcal{G} \oplus \mathcal{G}$. More precisely, if $\Lambda$ is such a subspace and $U_{\Lambda}$ is the Cayley transform of $\Lambda$ having the matrix $\left(u_{j k}\right)$ in the standard basis of $\mathcal{G}$, then the corresponding Schrödinger operator $H=H^{\Lambda}$ is defined on those functions $f \in \mathcal{D}\left(S^{*}\right)$ the components of which obey the boundary conditions

$$
\begin{gathered}
\sum_{k=1}^{n}\left[\left(\delta_{j k}-u_{j k}\right) b_{k}\left(f_{0}\right)+\left(\delta_{j, k+n}-u_{j, k+n}\right) f_{k}(0)\right]= \\
i \sum_{k=1}^{n}\left[\left(\delta_{j k}+u_{j k}\right) a_{k}\left(f_{0}\right)-\left(\delta_{j, k+n}+u_{j, k+n}\right) f_{k}^{\prime}(0)\right], \quad j=1, \ldots, 2 n .
\end{gathered}
$$

If $\Lambda$ is the graph of a self-adjoint operator $L$ in $\mathcal{G}$ with a Hermitian $2 n \times 2 n$-matrix $\left(\lambda_{j k}\right)$ then conditions (27) take a simpler form:

$$
\begin{aligned}
b_{j}\left(f_{0}\right) & =\sum_{k=1}^{n}\left[\lambda_{j, k} a_{k}\left(f_{0}\right)-\lambda_{j, k+n} f_{k}^{\prime}(0)\right], \\
f_{j}(0) & =\sum_{k=1}^{n}\left[\lambda_{j+n, k} a_{k}\left(f_{0}\right)-\lambda_{j+n, k+n} f_{k}^{\prime}(0)\right], \quad j=1, \ldots, n .
\end{aligned}
$$

In the general case there are a finite subset $\eta \subset\{1, \ldots, 2 n\}$ and a Hermitian $2 n \times 2 n$-matrix $L=\left(\lambda_{j k}\right)$ such that the conditions (27) take the following equivalent form:

$$
\begin{aligned}
& b_{j}\left(f_{0}\right)=\sum_{k \in \theta} \lambda_{j k} a_{k}\left(f_{0}\right)-\sum_{k \in \theta^{\prime}} \lambda_{j k} b_{k}\left(f_{0}\right)-\sum_{k \in \omega} \lambda_{j k} f_{k-n}^{\prime}(0)+\sum_{k \in \omega^{\prime}} \lambda_{j k} f_{k-n}(0), \quad j \in \theta ; \\
& a_{j}\left(f_{0}\right)=\sum_{k \in \theta} \lambda_{j k} a_{k}\left(f_{0}\right)-\sum_{k \in \theta^{\prime}} \lambda_{j k} b_{k}\left(f_{0}\right)-\sum_{k \in \omega} \lambda_{j k} f_{k-n}^{\prime}(0)+\sum_{k \in \omega^{\prime}} \lambda_{j k} f_{k-n}(0), \quad j \in \theta^{\prime} ;
\end{aligned}
$$




$$
\begin{aligned}
& f_{j}(0)=\sum_{k \in \theta} \lambda_{j+n, k} a_{k}\left(f_{0}\right)-\sum_{k \in \theta^{\prime}} \lambda_{j+n, k} b_{k}\left(f_{0}\right)-\sum_{k \in \omega} \lambda_{j+n, k} f_{k-n}^{\prime}(0)+\sum_{k \in \omega^{\prime}} \lambda_{j+n, k} f_{k-n}(0), \quad j+n \in \omega ; \\
& f_{j}^{\prime}(0)=\sum_{k \in \theta} \lambda_{j+n, k} a_{k}\left(f_{0}\right)-\sum_{k \in \theta^{\prime}} \lambda_{j+n, k} b_{k}\left(f_{0}\right)-\sum_{k \in \omega} \lambda_{j+n, k} f_{k-n}^{\prime}(0)+\sum_{k \in \omega^{\prime}} \lambda_{j+n, k} f_{k-n}(0), \quad j+n \in \omega^{\prime} ;
\end{aligned}
$$

where the sets $\theta, \theta^{\prime}, \omega$, and $\omega^{\prime}$ are defined in Remark 4.

Proof. The result follows immediately from Theorem A, Theorem 1, and Proposition C.

Below we collect the most interesting particular cases of Schrödinger operators on $\widehat{X}$ with given potentials. For this purpose we need some notions concerning point perturbations of Schrödinger operators on the manifold $X$. Let $B=\left(\beta_{j k}\right)$ be a Hermitian $n \times n$-matrix, $\theta$ a subset of $\{1, \ldots, n\}$, and $\theta^{\prime}=\{1, \ldots, n\} \backslash \theta$. Then the conditions

$$
\begin{gathered}
f_{0} \in \mathcal{D}\left(S_{0}^{*}\right) ; \\
b_{j}\left(f_{0}\right)=\sum_{k \in \theta} \beta_{j k} a_{k}\left(f_{0}\right)-\sum_{k \in \theta^{\prime}} \beta_{j k} b_{k}\left(f_{0}\right), \quad j \in \theta ; \\
a_{j}\left(f_{0}\right)=\sum_{k \in \theta} \beta_{j k} a_{k}\left(f_{0}\right)-\sum_{k \in \theta^{\prime}} \beta_{j k} b_{k}\left(f_{0}\right), \quad j \in \theta^{\prime} ;
\end{gathered}
$$

define a generic self-adjoint extension $H_{0}^{B, \theta}$ of the operator $S_{0}$. In particular, if $B=0$ and $\theta=\emptyset$, then $H_{0}^{B, \theta}$ is the Schrödinger operator $H_{0}$. If $\theta=\{1, \ldots, n\}$, then the operator $H_{0}^{B}=H_{0}^{B, \theta}$ is called a point perturbation of $H_{0}$ supported by the points $q_{1}, \ldots, q_{n}$ [2]. Generally speaking, this perturbation is non-local in the sense of [16]. If $B$ is a diagonal matrix, $\beta_{j k}=\beta_{j} \delta_{j k}, \beta_{j} \in \mathbb{R}$, then $H_{0}^{B}$ is called a local point perturbation of $H_{0}$.

In what follows we shall represent an arbitrary Hermitian $2 n \times 2 n$-matrix $L=\left(\lambda_{j k}\right)$ in block form:

$$
L=\left[\begin{array}{cc}
B & A \\
A^{*} & C
\end{array}\right],
$$

where $B=\left(\beta_{j k}\right)$ and $C=\left(\gamma_{j k}\right)$ are Hermitian $n \times n$-matrices whereas $A=\left(\alpha_{j k}\right)$ is an arbitrary complex $n \times n$-matrix.

Examples. We list four important particular cases of the Schrödinger operator $H$.

(1) Let $\eta=\emptyset$. Then the conditions (29) take the following simpler form:

$$
\begin{aligned}
a_{j}\left(f_{0}\right) & =-\sum_{k=1}^{n} \beta_{j k} b_{k}\left(f_{0}\right)+\sum_{k=1}^{n} \alpha_{j k} f_{k}(0), \\
f_{j}^{\prime}(0) & =-\sum_{k=1}^{n} \bar{\alpha}_{k j} b_{k}\left(f_{0}\right)+\sum_{k=1}^{n} \gamma_{j k} f_{k}(0), \quad j=1, \ldots, n .
\end{aligned}
$$


If $A=C=0$, then $H=H_{0}^{B, \emptyset} \oplus H_{1}^{\mathrm{N}} \oplus \ldots \oplus H_{n}^{\mathrm{N}}$. If, in addition, $B=0$, then

$$
a_{j}\left(f_{0}\right)=0, \quad f_{j}^{\prime}(0)=0, \quad j=1, \ldots, n,
$$

hence $H$ coincides with $H_{\mathrm{N}}$.

(2) Let $\eta=\{1, \ldots, 2 n\}$, then the conditions (29) take the form

$$
\begin{aligned}
b_{j}\left(f_{0}\right) & =\sum_{k=1}^{n} \beta_{j k} a_{k}\left(f_{0}\right)-\sum_{k=1}^{n} \alpha_{j k} f_{k}^{\prime}(0), \\
f_{j}(0) & =\sum_{k=1}^{n} \bar{\alpha}_{k j} a_{k}\left(f_{0}\right)-\sum_{k=1}^{n} \gamma_{j k} f_{k}^{\prime}(0), \quad j=1, \ldots, n,
\end{aligned}
$$

and we return to the conditions (28). We shall denote this operator by $H_{\mathrm{D}}^{L}$ and call it a Schrödinger operator of Dirichlet type. It is clear that this operator is disjoint from $H_{\mathrm{N}}$. If $A=C=0$, then $H_{\mathrm{D}}^{L}=S_{0}^{B} \oplus H_{1}^{\mathrm{D}} \oplus \ldots \oplus H_{n}^{\mathrm{D}}$.

(3) Let $\eta=\{n+1, \ldots, 2 n\}$. Then the conditions (29) become

$$
\begin{aligned}
a_{j}\left(f_{0}\right) & =-\sum_{k=1}^{n} \beta_{j k} b_{k}\left(f_{0}\right)-\sum_{k=1}^{n} \alpha_{j k} f_{k}^{\prime}(0), \\
f_{j}(0) & =-\sum_{k=1}^{n} \bar{\alpha}_{k j} b_{k}\left(f_{0}\right)-\sum_{k=1}^{n} \gamma_{j k} f_{k}^{\prime}(0), \quad j=1, \ldots, n .
\end{aligned}
$$

If $A=C=0$, then we get an operator $H=H_{0}^{B, \emptyset} \oplus H_{1}^{\mathrm{D}} \oplus \ldots \oplus H_{n}^{\mathrm{D}}$. If, in addition, $B=0$, then

$$
a_{j}\left(f_{0}\right)=0, \quad f_{j}(0)=0, \quad j=1, \ldots, n,
$$

i.e. $H$ coincides with $H_{\mathrm{D}}$.

(4) Let $\eta=\{1, \ldots, n\}$. Then the conditions (29) take the form

$$
\begin{aligned}
b_{j}\left(f_{0}\right) & =\sum_{k=1}^{n} \beta_{j k} a_{k}\left(f_{0}\right)+\sum_{k=1}^{n} \alpha_{j k} f_{k}(0), \\
f_{j}^{\prime}(0) & =\sum_{k=1}^{n} \bar{\alpha}_{k j} a_{k}\left(f_{0}\right)+\sum_{k=1}^{n} \gamma_{j k} f_{k}(0), \quad j=1, \ldots, n .
\end{aligned}
$$

We shall denote this operator by $H_{\mathrm{N}}^{L}$ and call it a Schrödinger operator of Neumann type. It is clear that this operator is disjoint from $H_{\mathrm{D}}$. If $A=C=0$, then $H_{\mathrm{N}}^{L}=H_{0}^{B} \oplus H_{1}^{\mathrm{N}} \oplus \ldots \oplus H_{n}^{\mathrm{N}}$. In the case $n=2$, the operator $H_{\mathrm{N}}^{L}$ has been considered in 24] and 46.

Theorem 1 implies the following description of the resolvents of Schrödinger operators.

Theorem 3. Let $\Lambda$ be a Lagrangian subspace of $\mathcal{G} \oplus \mathcal{G}$ and $H=H^{\Lambda}$ the Schrödinger operator defined by the boundary condition $\Gamma f \in \Lambda$. Then the resolvent $R(z)=(H-z)^{-1}$ of $H$ is given by the Krein formula

$$
R(z)=R_{\mathrm{N}}(z)-\gamma(z)[Q(z)-\Lambda]^{-1} \gamma^{*}(\bar{z})
$$


where $R_{\mathrm{N}}(z)=\left(H_{\mathrm{N}}-z\right)^{-1}$.

Similarly, if $H$ is defined by the boundary condition $\Gamma_{\mathrm{D}} f \in \Lambda$, then the resolvent $R(z)=(H-z)^{-1}$ is given by the expression

$$
R(z)=R_{\mathrm{D}}(z)-\gamma_{\mathrm{D}}(z)\left[Q_{\mathrm{D}}(z)-\Lambda\right]^{-1} \gamma_{\mathrm{D}}^{*}(\bar{z}),
$$

where $R_{\mathrm{D}}(z)=\left(H_{\mathrm{D}}-z\right)^{-1}$. In particular, if $H=H_{\mathrm{D}}^{L}\left(\right.$ respectively, $\left.H=H_{\mathrm{N}}^{L}\right)$, then (32) (respectively (33)) contains a single-valued operator $\Lambda$ with matrix $L$. In any case, using (8), we can rewrite (32) (or (33)) in the form

$$
R^{L, \eta}(z)=R_{\eta}(z)-\gamma_{\eta}(z)\left[Q_{\eta}(z)-L\right]^{-1} \gamma_{\eta}^{*}(\bar{z}),
$$

where $L$ is a Hermitian operator in $\mathcal{G}$.

\section{Spectral and scattering properties of the Schrödinger operator on a "hedgehog shaped" space}

From this section on we suppose that the manifold $X$ is compact. Therefore, the spectrum $\sigma\left(H_{0}\right)$ is discrete; let $\mu_{0}<\mu_{1}<\ldots<\mu_{m}<\ldots$ be the complete set of eigenvalues of $H_{0}$. We shall denote the eigenspace of $H_{0}$ corresponding to $\mu_{m}$ by $\mathcal{H}_{0}\left(\mu_{m}\right)$; in each $\mathcal{H}_{0}\left(\mu_{m}\right)$ we fix an orthonormal basis $\psi_{m}^{(1)}, \ldots, \psi_{m}^{\left(l_{m}\right)}$. Denote by $\sigma^{p}\left(H_{0}\right)$ the following subset of $\sigma\left(H_{0}\right)$ :

$$
\sigma^{p}\left(H_{0}\right):=\left\{\mu_{m} \in \sigma\left(H_{0}\right): \exists j \in\{1, \ldots, n\}, \exists \psi \in \mathcal{H}_{0}\left(\mu_{m}\right) \text { s.t. } \psi\left(q_{j}\right) \neq 0\right\} .
$$

Proposition 1. $Q_{0}(z)$ is a meromorphic matrix-valued function on the complex plain $\mathbb{C}$. The set of poles of $Q_{0}$ is infinite and coincides with $\sigma^{p}\left(H_{0}\right)$.

Proof. Using Mercer's Theorem it is not hard to derive the equality

$$
\frac{\partial\left[Q_{0}(z)\right]_{j k}}{\partial z}=\sum_{m=0}^{\infty}\left(\mu_{m}-z\right)^{-2} \sum_{s=1}^{l_{m}} \overline{\psi_{m}^{(s)}\left(q_{j}\right)} \psi_{m}^{(s)}\left(q_{k}\right),
$$

where the series converges absolutely and locally uniformly with respect to $z, z \in \mathbb{C} \backslash \sigma\left(H_{0}\right)$. It is hence clear that $Q_{0}$ is meromorphic and $\sigma^{p}\left(H_{0}\right)$ is the set of poles for $Q_{0}$. Suppose that this set is finite; then there exists $m_{0}$ such that $\mu_{m} \notin \sigma^{p}\left(H_{0}\right) \forall m>m_{0}$. Consider the linear hull $\mathcal{L}$ of all the eigenfunctions $\psi_{m}^{(s)}$, then $\mathcal{L} \subset C(X)$. Fix $j \in\{1, \ldots, n\}$ and set $q=q_{j}$. If $\varphi \in \mathcal{L}$, then the relations $\left\langle\psi_{m}^{(s)} \mid \varphi\right\rangle=0 \forall m \leq m_{0}, s=1, \ldots, l_{m}$, imply $\delta_{q}(\varphi):=\varphi(q)=0$. Therefore the linear functional $\delta_{q}$ on $\mathcal{L}$ is a linear combination of the linear functionals $\left\langle\psi_{m}^{(s)}\right|$ $\left(m \leq m_{0}, s=1, \ldots, l_{m}\right)$. Since $\mathcal{L}$ is dense in $C(X)$ with respect to both the Hilbert and Chebyshev norms, we conclude that $\delta_{q}$ is a continuous functional on $C(X)$ with respect to the topology induced from $L^{2}(X)$. This contradiction concludes the proof.

Remark 5. Generally speaking, the set $\sigma^{p}\left(H_{0}\right)$ depends on the tuple $\left(q_{1}, \ldots, q_{n}\right)$ but the set $Y=\left\{\left(q_{1}, \ldots q_{n}\right) \in X^{n}: \sigma^{p}\left(H_{0}\right)=\sigma\left(H_{0}\right)\right\}$ is generic both in sense of measure and category (i.e. the set $X^{n} \backslash Y$ is a zero-measure set of the first Baire class). Moreover, if $X$ is a homogeneous manifold, then $X=Y$ independently of the tuple $\left(q_{1}, \ldots, q_{n}\right)$. 
The structure of the spectrum for an arbitrary self-adjoint extension of the operator $S_{0}$ (in particular, for the point perturbation of $H_{0}$ ) is very simple. Namely, the following proposition is an evident consequence of theorems 14.9 and 14.10 from [58].

Proposition 2. Let $\widetilde{H}_{0}$ be a self-adjoint extension of $S_{0}$. Then $\widetilde{H}_{0}$ is bounded from below and the spectrum of $\widetilde{H}_{0}$ is purely discrete: $\sigma\left(\widetilde{H}_{0}\right)=\sigma_{\mathrm{dis}}\left(\widetilde{H}_{0}\right)$.

The spectral properties of a Schrödinger operator on $\widehat{X}$ are rather rich. Before we describe them, we settle the following notations. For the rest of this section $H$ will denote the Schrödinger operator on $\widehat{X}$ defined by a Schrödinger operator $H_{0}$ on $X$ and a Lagrangian subspace $\Lambda \subset \mathcal{G} \oplus \mathcal{G}$. The next theorem describes the spectral properties of $H$.

Theorem 4. The following assertions hold.

(i) $\sigma_{\mathrm{ess}}(H)=\sigma_{\mathrm{ac}}(H)=[0,+\infty)$;

(ii) $\sigma_{\mathrm{sc}}(H)=\emptyset$;

(iii) $\sigma_{\mathrm{dis}}(H)$ is a finite (possibly, empty) subset of $(-\infty, 0)$;

(iv) $\sigma_{\mathrm{pp}}(H) \cap[0,+\infty) \subset \sigma\left(\widetilde{H}_{0}\right)$, where $\widetilde{H}_{0}$ is a self-adjoint extension of $S_{0}$ (therefore, $\sigma_{\mathrm{pp}}(H)$ has no accumulation points);

(v) the multiplicity of an eigenvalue $E_{0} \in \sigma_{\mathrm{pp}}(H)$ does not exceed $2 n+m$, where $m$ is the multiplicity of $E_{0}$ in the spectrum of $H_{0}$. Moreover, let $N$ be the number of eigenvalues $E$ of $H$ (counting multiplicity) obeying the inequality $E<\min \left(0, \inf \sigma\left(H_{0}\right)\right)$; then $0 \leq N \leq 2 n$.

Proof. Clearly, the spectrum of $H_{\mathrm{N}}$ possesses all the properties (i)-(v). Therefore, general theorems about self-adjoint extensions with finite deficiency indices (Theorems 14.9 and 14.10 from [58], Theorem 18 from [50]) imply properties (iii) and (v) for the operator $H$. Furthermore, taking into account (32) we see that the equality $\sigma_{\text {ess }}(H)=[0,+\infty)$ follows from the Weyl theorem ([65], Theorem XII.14) and that the equality $\sigma_{\text {ac }}(H)=[0,+\infty)$ is a consequence of the Birman-Kuroda theorem ([64], Theorem XI.9).

Let us prove property (iv). Fix a representation of $H$ in the form $H^{L, \eta}$, where $\eta \subset\{1, \ldots, 2 n\}$ and $L$ is a Hermitian $2 n \times 2 n$-matrix. Let $E_{0}, E_{0} \geq 0$, be an eigenvalue of $H$ with an eigenvector $f=\left(f_{0}, f_{1}, \ldots, f_{n}\right)$. For every $j=1, \ldots, n$ the function $f_{j}$ belongs to $L^{2}\left(\mathbb{R}_{+}\right)$and obeys the equation $-f_{j}^{\prime \prime}=E_{0} f_{j}$; hence, $f_{j}=0$. Using the first two equations from (29) we show that $E_{0}$ is an eigenvalue of $\widetilde{H}_{0}=H_{0}^{B, \theta}$, where $\theta=\{1, \ldots, n\} \cap \eta$ and $B$ is related to $L$ by Eq. (31).

It remains to prove property (ii). Denote by $\mathcal{L}$ the dense subspace of all elements $f=\left(f_{0}, f_{1}, \ldots, f_{n}\right)$ from $\mathcal{H}$ such that $f_{0} \in C(X), f_{j} \in C_{0}^{\infty}(0,+\infty), j=1, \ldots, n$. Let $\mathcal{F}$ be a family of functions which are analytic in the upper half-plane $\mathbb{C}^{+}=\{z \in \mathbb{C}: \operatorname{Im} z>0\}$; we say that the family $\mathcal{F}$ is bounded near a point $E, E \in \mathbb{R}$, if there exists a neighborhood $V$ of $E$ such that every function from $\mathcal{F}$ is bounded in $V \cap \mathbb{C}^{+}$. According to Theorem XIII.20 from 65 it is enough to prove that for some countable subset $Z$ of $\mathbb{R}$ the family of the functions $z \mapsto\langle f \mid R(z) g\rangle$, where $f$ and $g$ run through $\mathcal{L}$, is bounded near every point $E$, $E \in(0,+\infty) \backslash Z$. It is clear that for $H=H_{\mathrm{N}}$ this family is bounded near the points from 
$(0,+\infty) \backslash \sigma\left(H_{0}\right)$. Moreover, let $\mathcal{F}$ be the family of functions of the form

$$
z \mapsto \int_{X} G_{0}\left(x, q_{j} ; z\right) f_{0}(x) d \lambda(x),
$$

or

$$
z \mapsto \int_{0}^{\infty} G_{j}(x, 0 ; z) f_{j}(x) d x,
$$

where $j=1, \ldots, n$ and $f=\left(f_{0}, f_{1}, \ldots, f_{n}\right) \in \mathcal{L}$. Then the family $\mathcal{F}$ is bounded near every point from $(0,+\infty) \backslash \sigma\left(H_{0}\right)$. According to (32) it remains to show that there exists a discrete subset $Z_{0} \subset \mathbb{R} \backslash \sigma\left(H_{0}\right)$ such that the elements of the matrix $[Q(z)-\Lambda]^{-1}$ form a bounded family near every point from $(0,+\infty) \backslash Z_{0}$. Rewrite $[Q(z)-\Lambda]^{-1}$ in the form $J_{\Lambda}\left[P_{\Lambda} Q(z) J_{\Lambda}-\right.$ $L]^{-1} P_{\Lambda}$ (see (7)). The elements of the matrix $Q(z)$ have analytic continuations from the halfplane $\mathbb{C}_{+}$to a neighborhood of the set $(0,+\infty) \backslash \sigma\left(H_{0}\right)$; moreover, $\operatorname{det}\left[P_{\Lambda} Q(z) J_{\Lambda}-L\right] \neq 0$, if $\operatorname{Im} z>0$. Therefore, we obtain the required property from standard analyticity arguments.

Now we are going to define the scattering matrix for the Schrödinger operator $H$ on $\widehat{X}$ following the ideas of geometric scattering theory (see, e.g. [54]). First of all we note that there exists a natural extension of $H$ to a domain of functions not belonging to $L^{2}(\widehat{X})$. Namely, Lemma 1 defines the boundary value operators $\Gamma_{j}^{(1)}$ and $\Gamma_{j}^{(2)}$ for every function from $H_{\mathrm{loc}}^{2}\left(\mathbb{R}_{+}^{(j)}\right)$. Therefore, (24) defines the operators $\Gamma_{j}$ and $\Gamma_{j}$ for every function $f=\left(f_{0}, f_{1}, \ldots, f_{n}\right)$ from $\mathcal{D}\left(S_{0}^{*}\right) \oplus H_{\text {loc }}^{2}\left(\mathbb{R}_{+}^{(1)}\right) \oplus \ldots \oplus H_{\text {loc }}^{2}\left(\mathbb{R}_{+}^{(n)}\right)$. Hence, we can consider the operator $H$ to be defined on the domain, $\mathcal{D}_{\text {loc }}(H)$, consisting of all functions $f$ from $\mathcal{D}\left(S_{0}^{*}\right) \oplus H_{\text {loc }}^{2}\left(\mathbb{R}_{+}^{(1)}\right) \oplus \ldots \oplus H_{\text {loc }}^{2}\left(\mathbb{R}_{+}^{(n)}\right)$ obeying the boundary condition $\left(\Gamma^{(1)} f, \Gamma^{(2)} f\right) \in \Lambda$ (this operator takes values in the space $\left.L_{\text {loc }}^{2}(\widehat{X})=L^{2}(X) \oplus L_{\text {loc }}^{2}\left(\mathbb{R}_{+}^{(1)}\right) \oplus \ldots \oplus L_{\text {loc }}^{2}\left(\mathbb{R}_{+}^{(n)}\right)\right)$. If $H$ is represented in the form $H=H^{L, \eta}$, then the last condition may be replaced by condition (29). To define the scattering matrix we need solutions to the Schrödinger equation

$$
H f=k^{2} f
$$

$f \in \mathcal{D}_{\text {loc }}(H), k \geq 0$, the so-called scattering states, which have a special behavior in the channels $\mathbb{R}_{+}^{(j)}$. The following theorem provides us with such solutions.

Theorem 5 (Existence and uniqueness of scattering states). For every Schrödinger operator $H=H^{\Lambda}$ on $\widehat{X}$ there exists a discrete subset $Z_{H}$ of $\mathbb{R}$ such that the following assertion is valid.

For a given $j \in\{1, \ldots, n\}$ and every $k>0, k^{2} \notin Z_{H}$, the Schrödinger equation (35) has a unique solution $f=\left(f_{0}, f_{1}, \ldots, f_{n}\right)$ satisfying the conditions:

(i) $f_{j}(x)=\exp (-i k x)+r_{j}(k) \exp (i k x)$,

(ii) if $l \in\{1, \ldots, n\}$ and $l \neq j$, then $f_{l}(x)=t_{l j}(k) \exp (i k x)$,

where $r_{j}(k), t_{l j}(k) \in \mathbb{C}$.

Proof. We define $Z_{H}$ as the union of the following sets: 1) $\sigma\left(H_{0}\right)$;2) $\sigma\left(H_{0}^{B, \theta}\right)$ if $H$ may be represented in the form $H=H^{L, \eta}$ and $(B, \theta)$ is related to $(L, \eta)$ with (25), (31); 3) the set of 
all solutions to the equation $\operatorname{det}\left[P_{\Lambda} Q(E) J_{\Lambda}-L\right]=0$ where $Q(E)$ is the analytic continuation of $Q(z)$ from the upper half-plane $\mathbb{C}^{+}$to $\mathbb{R}^{+}$(see the proof of Theorem 4 ). Clearly, $Z_{H}$ is discrete.

Further we note that for every $\zeta=\left(\zeta_{l}\right)_{1 \leq l \leq 2 n} \in \mathbb{C}^{2 n}=\mathcal{G}$ the function $\gamma(z) \zeta=$ $\left(\varphi_{0}, \varphi_{1}, \ldots, \varphi_{n}\right)$ has the form

$$
\begin{gathered}
\varphi_{0}(x)=\sum_{m=1}^{n} \zeta_{m} G_{0}\left(x, q_{m} ; z\right), \\
\varphi_{l}(x)=\frac{\zeta_{l+n}}{\sqrt{-z}} \exp (-\sqrt{-z} x), \quad 0<l \leq n,
\end{gathered}
$$

(see (10)), (23), and (24)). Therefore, for any $\varphi \in \mathcal{H}$

$$
\gamma^{*}(\bar{z}) \varphi=\left(\zeta_{l}\right)_{1 \leq l \leq 2 n}
$$

where

$$
\begin{gathered}
\zeta_{l}=\int_{X} G_{0}\left(q_{l}, x ; z\right) \varphi_{0}(x) d \lambda(x), \quad 1 \leq l \leq n ; \\
\zeta_{l}=\frac{1}{\sqrt{-z}} \int_{0}^{\infty} \exp (-\sqrt{-z} x) \varphi_{l-n}(x) d x, \quad n+1 \leq l \leq 2 n .
\end{gathered}
$$

Fix now $k>0, k^{2} \notin Z_{H}$, and put $z=k^{2}+i \varepsilon$, where $0<\varepsilon \leq \varepsilon_{0}$, with some $\varepsilon_{0}>0$. It is clear that an element $g$ from $\mathcal{H}$ belongs to $\mathcal{D}(H)$ if and only if $g=R(z) h$, where $h$ is an element from $\mathcal{H}$ (which depends on $z$ ). Set $\psi=R_{\mathrm{N}}(z) h$, then $\psi \in \mathcal{D}\left(H_{\mathrm{N}}\right)$ and from (32)

$$
g=\psi-\gamma(z)[Q(z)-\Lambda]^{-1} \gamma^{*}(\bar{z})\left(H_{\mathrm{N}}-z\right) \psi
$$

and

$$
(H-z) g=\left(H_{\mathrm{N}}-z\right) \psi \text {. }
$$

Conversely, every function $\psi \in \mathcal{D}\left(H_{\mathrm{N}}\right)$ defines, by (40), an element $g$ from $\mathcal{D}(H)$ in such a way that (41) holds. Note that according to (38) and (39), the vector $\xi=\gamma^{*}(\bar{z})\left(H_{\mathrm{N}}-z\right) \psi$ has the form

$$
\xi_{l}= \begin{cases}\psi_{0}\left(q_{l}\right), & \text { if } 1 \leq l \leq n \\ \psi_{l-n}(0), & \text { if } n+1 \leq l \leq 2 n .\end{cases}
$$

Therefore, we can rewrite (40) as

$$
g=\psi-\gamma(z) \zeta(\psi)
$$

with

$$
\zeta_{l}(\psi)=\sum_{m=1}^{n}[Q(z)-\Lambda]_{l m}^{-1} \psi_{0}\left(q_{m}\right)+\sum_{m=n+1}^{2 n}[Q(z)-\Lambda]_{l m}^{-1} \psi_{m-n}(0)
$$


Now fix $j \in\{1, \ldots, n\}$ and define $\psi=\left(\psi_{0}, \psi_{1}, \ldots, \psi_{n}\right)$ from $L_{\text {loc }}^{2}(\widehat{X})$ by

$$
\psi_{l}(x)= \begin{cases}\exp (i k x)+\exp (-i k x), & \text { if } l=j, \\ 0, & \text { otherwise }\end{cases}
$$

It is clear that $\psi \in \mathcal{D}_{\text {loc }}\left(H_{\mathrm{N}}\right)$ but $\psi \notin \mathcal{D}\left(H_{\mathrm{N}}\right)$. To obtain a function from $\mathcal{D}(H)$ we choose for $a>0$ a cut-off function $\chi_{a} \in C_{0}^{\infty}\left(\mathbb{R}_{+}\right)$such that $\chi_{a}(x)=1$ if $0 \leq x \leq a, \chi_{a}(x)=0$ if $x>a+1$, and $0 \leq \chi_{a}(x) \leq 1 \forall x \in \mathbb{R}_{+}$. Set $\widetilde{\chi}_{a}:=\left(1, \chi_{a}, \ldots, \chi_{a}\right)$, it is clear that the product $\tilde{\chi}_{a} \psi=\left(\psi_{0}, \chi_{a} \psi_{1}, \ldots, \chi_{a} \psi_{n}\right)$ is in $\mathcal{D}\left(H_{\mathrm{N}}\right)$, and hence defines a function

$$
g^{(a)}:=\widetilde{\chi}_{a} \psi-\gamma(z) \zeta\left(\widetilde{\chi}_{a} \psi\right)
$$

in $\mathcal{D}(H)$ such that

$$
(H-z) g^{(a)}=\left(H_{\mathrm{N}}-z\right) \widetilde{\chi}_{a} \psi .
$$

We write the matrix $[Q(z)-\Lambda]^{-1}$ in block form,

$$
[Q(z)-\Lambda]^{-1}=\left[\begin{array}{cc}
N(z) & W(z) \\
M(z) & V(z)
\end{array}\right]
$$

where $W(z)=\left(w_{l m}(z)\right)$ and $V(z)=\left(v_{l m}(z)\right)$ are $n \times n$-matrices. From (42) we have

$$
\begin{gathered}
\zeta_{l}\left(\widetilde{\chi}_{a} \psi\right)=\sum_{m=n+1}^{2 n}[Q(z)-\Lambda]_{l, m}^{-1} \psi_{m-n}(0) \\
=2 \sum_{m=1}^{n}[Q(z)-\Lambda]_{l, m+n}^{-1} \delta_{j m}=2[Q(z)-\Lambda]_{l, j+n}^{-1} .
\end{gathered}
$$

In other words,

$$
\zeta_{l}\left(\widetilde{\chi}_{a} \psi\right)= \begin{cases}2 w_{l j}(z), & \text { if } 1 \leq l \leq n, \\ 2 v_{l j}(z), & \text { if } n+1 \leq l \leq 2 n .\end{cases}
$$

Hence, from (36), (37), and (44) we get

$$
\begin{gathered}
g_{0}^{(a)}(x)=-2 \sum_{m=1}^{n} w_{m j}(z) G_{0}\left(x, q_{m} ; z\right), \\
g_{l}^{(a)}(x)=\delta_{l j} \chi_{a}(x) \psi_{j}(x)-\frac{2 v_{l j}(z)}{\sqrt{-z}} \exp (-\sqrt{-z} x), \quad 0<l \leq n .
\end{gathered}
$$

with

Passing to the limit $a \rightarrow \infty$ in (47) and (48), we obtain $g=\left(g_{0}, g_{1}, \ldots, g_{n}\right) \in L_{\mathrm{loc}}^{2}(\widehat{X})$

$$
\begin{gathered}
g_{0}(x)=-2 \sum_{m=1}^{n} w_{m j}(z) G_{0}\left(x, q_{m} ; z\right), \\
g_{l}(x)=\delta_{l j} \psi_{j}(x)-\frac{2 v_{l j}(z)}{\sqrt{-z}} \exp (-\sqrt{-z} x), \quad 0<l \leq n .
\end{gathered}
$$


Moreover, since $\Gamma^{(1)} g^{(a)}$ and $\Gamma^{(2)} g^{(a)}$ are independent of $a, g$ satisfies the boundary condition $\left(\Gamma^{(1)} g, \Gamma^{(2)} g\right) \in \Lambda$, and from (45) we have

$$
(H-z) g=\left(H_{\mathrm{N}}-z\right) \psi .
$$

In the limit $\varepsilon \rightarrow 0$ we have $\sqrt{-z} \rightarrow-i k$, whereas $g$ has a limit $f$ in $L_{\text {loc }}^{2}(\widehat{X})$ such that

$$
\begin{aligned}
f_{0}(x) & =-2 \sum_{m=1}^{n} w_{m j}\left(k^{2}\right) G_{0}\left(x, q_{m} ; k^{2}\right), \\
f_{l}(x) & =\delta_{l j} \psi_{j}(x)+\frac{2 v_{l j}\left(k^{2}\right)}{i k} \exp (i k x), \quad 0<l \leq n,
\end{aligned}
$$

since $k^{2} \notin \sigma\left(H_{0}\right)$. Moreover, in the sense of distributions,

$$
\begin{gathered}
(H-z) g \rightarrow\left(H-k^{2}\right) f, \\
\left(H_{\mathrm{N}}-z\right) \psi \rightarrow\left(H_{\mathrm{N}}-k^{2}\right) \psi=0 .
\end{gathered}
$$

Hence, $\left(H-k^{2}\right) f=0$. Further, $f$ satisfies the boundary condition $\left(\Gamma^{(1)} f, \Gamma^{(2)} f\right) \in \Lambda$. Indeed, since $k^{2} \notin \sigma\left(H_{0}\right)$ we have

$$
a_{m}\left(G_{0}\left(\cdot, q_{l} ; z\right)\right) \rightarrow \delta_{l m}, \quad b_{m}\left(G_{0}\left(\cdot, q_{l} ; z\right)\right) \rightarrow Q_{0}^{m l}\left(k^{2}\right)
$$

as $z \rightarrow k^{2}$ (see (14) and (16)). On the other hand, direct calculations show that $\Gamma_{l}^{(1)} g_{l} \rightarrow$ $\Gamma_{l}^{(1)} f_{l}$ and $\Gamma_{l}^{(2)} g_{l} \rightarrow \Gamma_{l}^{(2)} f_{l}$ as $z \rightarrow k^{2}(l=1, \ldots, n ;)$. Finally, from (49) we get the properties (i) and (ii) with

$$
\begin{aligned}
r_{j}(k) & =1-2 i k^{-1} v_{j j}\left(k^{2}\right), \\
t_{l j}(k) & =-2 i k^{-1} v_{l j}\left(k^{2}\right) .
\end{aligned}
$$

The proof is completed by establishing the uniqueness of $f$ which follows from Lemma 7 below.

Lemma 7. Let $f$ be a solution to the Schrödinger equation (35) for some $k^{2} \notin Z_{H}$, with the property that for all $l, 1 \leq l \leq n$,

$$
f_{l}(x)=\alpha_{l} \exp (i k x)
$$

for some $\alpha_{l} \in \mathbb{C}$. Then $f=0$.

Proof. Take $z_{\varepsilon}=k^{2}+i \varepsilon$ and $a>0$ as in the proof of the theorem. It is evident that $\tilde{\chi}_{a} f \in \mathcal{D}(H)$; denote

$$
\psi^{(\varepsilon)}=R_{\mathrm{N}}\left(z_{\varepsilon}\right)\left(H-z_{\varepsilon}\right) \tilde{\chi}_{a} f .
$$

Then

$$
\left(H_{\mathrm{N}}-z_{\varepsilon}\right) \psi^{(\varepsilon)}=\left(H-z_{\varepsilon}\right) \widetilde{\chi}_{a} f,
$$

and

$$
\tilde{\chi}_{a} f=\psi^{(\varepsilon)}-\gamma\left(z_{\varepsilon}\right) \zeta\left(\psi^{(\varepsilon)}\right) .
$$


Consider the integral kernel $G_{l}^{\mathrm{N}}(x, y ; z)$ of the operator $R_{l}^{\mathrm{N}}(z)=\left(H_{l}^{\mathrm{N}}-z\right)^{-1}$, then

$$
G_{l}^{\mathrm{N}}\left(x, y ; z_{\varepsilon}\right) \rightarrow \frac{i}{2 k}[\exp (i k|x-y|)+\exp (i k(x+y))] \equiv G_{l}^{\mathrm{N}}\left(x, y ; k^{2}\right)
$$

as $\varepsilon \rightarrow 0$ (see (11)). Denote by $\mathcal{H}_{l}^{(a)}$ the following subspace of $\mathcal{H}_{l}=L^{2}\left(\mathbb{R}_{+}^{(l)}\right)$ :

$$
\mathcal{H}_{l}^{(a)}:=\left\{\varphi \in L^{2}\left(\mathbb{R}_{+}^{(l)}\right): \operatorname{supp} \varphi \subset[0, a+1]\right\}
$$

then $G_{l}^{\mathrm{N}}(x, y ; z)$ is the kernel of a continuous linear operator from $\mathcal{H}_{l}^{(a)}$ to $L_{\text {loc }}^{2}\left(\mathbb{R}_{+}^{(l)}\right)$ (recall that $L_{\text {loc }}^{2}\left(\mathbb{R}_{+}^{(l)}\right)$ is endowed with the topology of $L^{2}$-convergence on compact subsets of $\left.\mathbb{R}_{+}^{(l)}\right)$. Set $\mathcal{H}^{(a)}:=\mathcal{H}_{0} \oplus \mathcal{H}_{1}^{(a)} \oplus \ldots \oplus \mathcal{H}_{n}^{(a)}$, then

$$
R_{\mathrm{N}}\left(k^{2}\right):=R_{0}\left(k^{2}\right) \oplus R_{1}^{\mathrm{N}}\left(k^{2}\right) \oplus \ldots \oplus R_{n}^{\mathrm{N}}\left(k^{2}\right)
$$

is a continuous linear operator from $\mathcal{H}^{(a)}$ to $L_{\text {loc }}^{2}(\widehat{X})$. Moreover, if $h \in \mathcal{H}^{(a)}$ then $R_{\mathrm{N}}\left(z_{\varepsilon}\right) h \rightarrow$ $R_{\mathrm{N}}\left(k^{2}\right) h$ as $\varepsilon \rightarrow 0$; in particular $\psi^{(\varepsilon)} \rightarrow R_{\mathrm{N}}\left(k^{2}\right)\left(H-k^{2}\right) \widetilde{\chi}_{a} f=: \psi^{(0)}$. Hence, $\widetilde{\chi}_{a} \psi^{(\varepsilon)} \rightarrow \widetilde{\chi}_{a} \psi^{(0)}$ in $L^{2}(\widehat{X})$ as $\varepsilon \rightarrow 0$, too. Fix $\varepsilon_{0}>0$ and put $z_{0}=k^{2}+i \varepsilon_{0}$, then

$$
\begin{gathered}
\left(H_{\mathrm{N}}-z_{0}\right) \psi^{(\varepsilon)}=\left(H_{\mathrm{N}}-z_{\varepsilon}\right) \psi^{(\varepsilon)}+\left(z_{\varepsilon}-z_{0}\right) \psi^{(\varepsilon)}= \\
\left(H-z_{\varepsilon}\right) \tilde{\chi}_{a} f+\left(z_{\varepsilon}-z_{0}\right) \psi^{(\varepsilon)} .
\end{gathered}
$$

Therefore, $\left(H_{\mathrm{N}}-z_{0}\right) \psi^{(\varepsilon)}$ has a limit in $L_{\text {loc }}^{2}(\widehat{X})$ as $\varepsilon \rightarrow 0$. Consequently,

$$
\psi_{l}^{(\varepsilon)}(x) \rightarrow \psi_{l}^{(0)}(x), \quad \frac{d}{d x} \psi_{l}^{(\varepsilon)}(x) \rightarrow \frac{d}{d x} \psi_{l}^{(0)}
$$

locally uniformly on $\mathbb{R}_{+}^{(l)}$ for each $l, 1 \leq l \leq n$. Now we have

$$
\begin{gathered}
\left(H_{\mathrm{N}}-z_{0}\right) \tilde{\chi}_{a} \psi^{(\varepsilon)}= \\
\widetilde{\chi}_{a}\left(H_{\mathrm{N}}-z_{0}\right) \psi^{(\varepsilon)}-2\left(0, \chi_{a}^{\prime}\left(\psi_{1}^{(\varepsilon)}\right)^{\prime}, \ldots, \chi_{a}^{\prime}\left(\psi_{n}^{(\varepsilon)}\right)^{\prime}\right)-\left(0, \chi_{a}^{\prime \prime} \psi_{1}^{(\varepsilon)}, \ldots, \chi_{a}^{\prime \prime} \psi_{n}^{(\varepsilon)}\right) .
\end{gathered}
$$

It follows from (54) that also $\left(H_{\mathrm{N}}-z_{0}\right) \widetilde{\chi}_{a} \psi^{(\varepsilon)}$ has a limit in $L^{2}(\widehat{X})$, and therefore, $\widetilde{\chi}_{a} \psi^{(\varepsilon)}$ has a limit in the graph topology of $\mathcal{D}\left(H_{\mathrm{N}}\right)$ as $\varepsilon \rightarrow 0$. Thus, $\tilde{\chi}_{a} \psi^{(0)} \in \mathcal{D}\left(H_{\mathrm{N}}\right)$ and $\left(H_{\mathrm{N}}-z_{0}\right) \widetilde{\chi}_{a} \psi^{(\varepsilon)} \rightarrow$ $\left(H_{\mathrm{N}}-z_{0}\right) \widetilde{\chi}_{a} \psi^{(0)}$ in $L^{2}(\widehat{X})$.

Now (52) implies that

$$
\begin{gathered}
\left(H_{\mathrm{N}}-z_{\varepsilon}\right) \widetilde{\chi}_{a} \psi^{(\varepsilon)}= \\
\widetilde{\chi}_{a}\left(H-z_{\varepsilon}\right) \widetilde{\chi}_{a} f-2\left(0, \chi_{a}^{\prime}\left(\psi_{1}^{(\varepsilon)}\right)^{\prime}, \ldots, \chi_{a}^{\prime}\left(\psi_{n}^{(\varepsilon)}\right)^{\prime}\right)-\left(0, \chi_{a}^{\prime \prime} \psi_{1}^{(\varepsilon)}, \ldots, \chi_{a}^{\prime \prime} \psi_{n}^{(\varepsilon)}\right) .
\end{gathered}
$$

Since $\left(\widetilde{\chi}_{a}\left(H-k^{2}\right) \widetilde{\chi}_{a} f\right)(x)=0$ if $x \in \mathbb{R}_{+}^{(l)}$ and $0 \leq x \leq a$, we get from (55) by passing to the limit $\varepsilon \rightarrow 0$

$$
\left(H_{\mathrm{N}}-\lambda\right) \widehat{\chi}_{a} \psi^{(0)}(x)=0, \quad \text { if } x \in \mathbb{R}_{+}^{(l)}, \quad 0 \leq x \leq a .
$$

Because $\widetilde{\chi}_{a} \psi^{(0)} \in \mathcal{D}\left(H_{\mathrm{N}}\right)$, we have for every $l \geq 1$ :

$$
\psi_{l}^{(0)}(x)=c_{l}(\exp (i k x)+\exp (-i k x)), \quad \text { if } x \in[0, a]
$$


Now, we turn to (53); for $l \geq 1$ this equality reads

$$
\chi_{a}(x) f_{l}(x)=\psi_{l}^{(\varepsilon)}(x)-\frac{\zeta_{l+n}\left(\psi^{(\varepsilon)}\right)}{\sqrt{-z}} \exp (-\sqrt{-z} x)
$$

where

$$
\zeta_{l+n}\left(\psi^{(\varepsilon)}\right)=\sum_{m=1}^{n}[Q(z)-\Lambda]_{l+n, m}^{-1} \psi_{0}^{(\varepsilon)}\left(q_{m}\right)+\sum_{m=n+1}^{2 n}[Q(z)-\Lambda]_{l+n, m}^{-1} \psi_{m-n}^{(\varepsilon)}(0)
$$

(see (37) and (42)). By definition of $\psi^{(\varepsilon)}$ we have $\psi_{0}^{(\varepsilon)}=R_{0}\left(k^{2}+i \varepsilon\right)\left(S_{0}^{*}-k^{2}-i \varepsilon\right) f_{0}$. Moreover $R_{0}(z)$ is a continuous mapping from $L^{2}(X)$ to $\mathcal{D}\left(H_{0}\right)$ endowed with the graph topology and hence, a continuous mapping from $L^{2}(X)$ to $C(X)$ which continuously depends on $\varepsilon \in\left[0, \varepsilon_{0}\right]$. Therefore, $\psi_{0}^{(\varepsilon)}\left(q_{m}\right) \rightarrow \psi_{0}^{(0)}\left(q_{m}\right)$ as $\varepsilon \rightarrow 0$. Hence, we obtain from (56) that for $l \geq 1$

$$
\chi_{a}(x) f_{l}(x)=\psi_{l}^{(0)}(x)+\frac{\zeta_{l+n}\left(\psi^{(0)}\right)}{i k} \exp (i k x)
$$

where the coefficients

$$
\zeta_{l+n}\left(\psi^{(0)}\right)=\sum_{m=1}^{n}\left[Q\left(k^{2}\right)-\Lambda\right]_{l+n, m}^{-1} \psi_{0}^{(0)}\left(q_{m}\right)+\sum_{m=n+1}^{2 n}\left[Q\left(k^{2}\right)-\Lambda\right]_{l+n, m}^{-1} \psi_{m-n}^{(0)}(0)
$$

are well defined because $k^{2} \notin Z_{H}$. Moreover, $\psi_{0}^{(0)}(q)=0$, since $k^{2} \notin \sigma\left(H_{0}\right)$, and (57) implies that for $x \in[0, a]$ and $l \geq 1$ the functions $f_{l}$ have the form

$$
f_{l}(x)=c_{l} \exp (-i k x)+c_{l}^{\prime} \exp (i k x)
$$

Comparing with (51), we get $c_{l}=0$ and hence, $\psi_{l}^{(0)}(x)=0$ for $x \in[0, a]$. Returning to (57) and (58) we obtain that $f_{l}(x)=0$ for $x \in[0, a]$. Since $a$ is arbitrary, $f_{l}=0 \forall l=1, \ldots, n$.

Using (29) we see that $f_{0}$ satisfies the boundary conditions (30). Moreover, by the hypothesis of the lemma, $f_{0}$ is a solution to the equation $\left(S_{0}^{*}-k^{2}\right) f_{0}=0$. Since $k^{2} \notin \sigma\left(H_{0}^{B, \theta}\right)$, we get $f_{0}=0$. Thus, the lemma is proven, and the proof of Theorem 5 is completed.

Property (i) of Theorem 5 means that the function $f_{j}(x)$ represents a superposition of an incoming wave $\exp (-i k x)$ and a reflected wave $r_{j}(k) \exp (i k x)$ in the channel $\mathbb{R}_{+}^{(j)}$.

Definition. $r_{j}(k)$ is called the reflection amplitude for $H$ in the channel $\mathbb{R}_{+}^{(j))}$ at energy $E=k^{2}$. The quantity $R_{j}(k)=\left|r_{j}(k)\right|^{2}$ is called the reflection coefficient (or the reflection probability) in the channel $\mathbb{R}_{+}^{(j)}$.

Condition (ii) in Theorem 5 means that the function $f_{l}(x)(l \neq j)$ represents an outgoing wave $t_{l j}(k) \exp (i k x)$ in the channel $\mathbb{R}_{+}^{(l)}$.

Definition. $t_{l j}(k)$ is called the transmission amplitude for $H$ from the channel $\mathbb{R}_{+}^{(j)}$ to the channel $\mathbb{R}_{+}^{(l)}$ at energy $E=k^{2}$. The quantity $T_{l j}(k)=\left|t_{l j}(k)\right|^{2}$ is called the transmission coefficient (or the transmission probability) from $\mathbb{R}_{+}^{(j)}$ to $\mathbb{R}_{+}^{(l)}$. 
Set

$$
s_{l j}(k)= \begin{cases}r_{j}(k), & \text { if } l=j \\ t_{l j}(k), & \text { otherwise } .\end{cases}
$$

The matrix $\Sigma(k)=\left(s_{l j}(k)\right)_{1 \leq l, j \leq n}$ is called the scattering matrix for $H$. We stress that $\Sigma(k)$ is defined for all $k>0$ with the exception of a discrete subset $Z_{H}$ of $\mathbb{R}$.

Theorem 6. The scattering matrix $\Sigma(k)$ is unitary for all $k \in Z_{H}$. If the matrix $\left[Q\left(k^{2}\right)-\Lambda\right]^{-1}$ is represented in the form (46), then

$$
\Sigma(k)=I-2 i k^{-1} V\left(k^{2}\right) .
$$

If $\Lambda$ is the graph of a Hermitian operator $L$ in $\mathcal{G}$ and the matrix $L$ is represented in the form (31), then

$$
\Sigma(k)=\left[C+A^{*}\left(Q_{0}\left(k^{2}\right)-B\right)^{-1} A+i k^{-1} I\right]\left[C+A^{*}\left(Q_{0}\left(k^{2}\right)-B\right)^{-1} A-i k^{-1} I\right]^{-1} .
$$

In particular, if the matrix $A$ is invertible, and $C$ is a scalar matrix (i.e. $C=\gamma I, \gamma \in \mathbb{R}$ ), then

$$
\begin{gathered}
\Sigma(k)=\left[i k I+(i k \gamma-1) A^{-1}\left(Q_{0}\left(k^{2}\right)-B\right) A^{*-1}\right]\left[i k I+(i k \gamma+1) A^{-1}\left(Q_{0}\left(k^{2}\right)-B\right) A^{*-1}\right]^{-1}= \\
A^{-1}\left[i k A A^{*}+(i k \gamma-1)\left(Q_{0}\left(k^{2}\right)-B\right)\right]\left[i k A A^{*}+(i k \gamma+1)\left(Q_{0}\left(k^{2}\right)-B\right)\right]^{-1} A .
\end{gathered}
$$

Proof. (59) follows immediately from (50) as obtained in the proof of Theorem 5. To get (60) we use the Frobenius formula for the inverse of a block-matrix [44]:

$$
\begin{gathered}
{\left[\begin{array}{rr}
A_{11} & A_{12} \\
A_{21} & A_{22}
\end{array}\right]^{-1}=} \\
{\left[\begin{array}{ll}
{\left[A_{11}-A_{12} A_{22}^{-1} A_{21}\right]^{-1}} & A_{11}^{-1} A_{12}\left[A_{21} A_{11}^{-1} A_{12}-A_{22}\right]^{-1} \\
{\left[A_{21} A_{11}^{-1} A_{12}-A_{22}\right]^{-1} A_{21} A_{11}^{-1}} & {\left[A_{22}-A_{21} A_{11}^{-1} A_{12}\right]^{-1}}
\end{array}\right]}
\end{gathered}
$$

Since

$$
Q(z)-L=\left[\begin{array}{cc}
Q_{0}(z)-B & -A \\
-A^{*} & i k^{-1} I-C
\end{array}\right]
$$

then

$$
V\left(k^{2}\right)=\left[i k^{-1} I-C-A^{*}\left(Q_{0}\left(k^{2}\right)-B\right)^{-1} A\right]^{-1},
$$

and hence

$$
\Sigma(k)=I-2\left[I+i k\left(C+A^{*}\left(Q_{0}\left(k^{2}\right)-B\right)^{-1} A\right)\right]^{-1} .
$$

Now we get (60) from (63) after some elementary algebra, (61) is an evident consequence of (60). 
In particular, (60) shows that $\Sigma(k)$ is the Cayley transform of the Hermitian matrix $C+A^{*}\left(Q_{0}\left(k^{2}\right)-B\right)^{-1} A$. Hence, in case $\Lambda$ is the graph of an Hermitian operator in $\mathcal{G}, \Sigma(k)$ is a unitary matrix. To prove the general case we use Proposition E.

In the notation of Example (2) from Section 2, (60) gives the scattering matrix for the Schrödinger operator of Dirichlet type, $H_{\mathrm{D}}^{L}$. We now derive an explicit expression for the scattering matrix in case of an arbitrary Schrödinger operator $H^{\Lambda}$. It is convenient to write the boundary conditions in the form (3) where the $(2 n \times 2 n)$-matrices $L$ and $M$ have block structure

$$
L=\left[\begin{array}{ll}
B & A_{1} \\
A_{2} & C
\end{array}\right], \quad M=\left[\begin{array}{ll}
Y & X_{1} \\
X_{2} & Z
\end{array}\right]
$$

and satisfy conditions (a) and (b) from Proposition B. In particular, condition (a) is equivalent to the relations

$$
\begin{aligned}
& B Y^{*}+A_{1} X_{1}^{*}=Y B^{*}+X_{1} A_{1}^{*}, \\
& B X_{2}^{*}+A_{1} Z^{*}=Y A_{2}^{*}+X_{1} C^{*}, \\
& A_{2} X_{2}^{*}+C Z^{*}=X_{2} A_{2}^{*}+Z C^{*} .
\end{aligned}
$$

Suppose for the moment that $M$ is invertible, then condition (3) reads: $\Gamma^{(2)} x=$ $\Lambda \Gamma^{(1)} x$ where $\Lambda=M^{-1} L$. Therefore, using the Frobenius formula (62) we obtain the following expression for the matrix $V$ from (46):

$$
\begin{gathered}
V=\left[i k^{-1} Z-C-\left(X_{2} Q_{0}-A_{2}\right)\left(Y Q_{0}-B\right)^{-1}\left(i k^{-1} X_{1}-A_{1}\right)\right]^{-1} \\
\cdot\left[Z-\left(X_{2} Q_{0}-A_{2}\right)\left(Y Q_{0}-B\right)^{-1} X_{1}\right] .
\end{gathered}
$$

Substituting (56) in (59) we finally obtain

$$
\begin{gathered}
\Sigma(k)=\left[i k C+Z-\left(X_{2} Q_{0}\left(k^{2}\right)-A_{2}\right)\left(Y Q_{0}\left(k^{2}\right)-B\right)^{-1}\left(i k A_{1}+X_{1}\right)\right]^{-1} \\
\cdot\left[i k C-Z-\left(X_{2} Q_{0}\left(k^{2}\right)-A_{2}\right)\left(Y Q_{0}\left(k^{2}\right)-B\right)^{-1}\left(i k A_{1}-X_{1}\right)\right] .
\end{gathered}
$$

Since invertible matrices are dense in the space of all $(2 n \times 2 n)$-matrices, expression (67) is valid for all boundary conditions of the form (3). In particular, if $M=I$ then we recover (60).

Next we consider some particular cases of (67) and determine the scattering matrices for the Schrödinger operators from Examples (1), (3), and (4) of Section 2 (Example (2) contains operators of Dirichlet type which are covered by (60)).

1. Consider Example (1) from Section 2. Using the notations there we have

$$
L=\left[\begin{array}{ll}
I & 0 \\
0 & I
\end{array}\right], \quad M=\left[\begin{array}{cc}
-B & A \\
A^{*} & -C
\end{array}\right] .
$$

Therefore,

$$
\Sigma(k)=\left[i k+C-A^{*}\left(Q_{0}^{-1}\left(k^{2}\right)+B\right)^{-1} A\right]\left[i k-C+A^{*}\left(Q_{0}^{-1}\left(k^{2}\right)+B\right)^{-1} A\right]^{-1} .
$$


2. Now we turn to Example (3) from Section 2. In this case

$$
L=\left[\begin{array}{cc}
-I & A \\
0 & C
\end{array}\right], \quad M=\left[\begin{array}{cc}
B & 0 \\
A^{*} & I
\end{array}\right]
$$

Hence

$$
\Sigma(k)=\left[C+i k^{-1}-A^{*}\left(Q_{0}^{-1}\left(k^{2}\right)+B\right)^{-1} A\right]\left[C-i k^{-1}-A^{*}\left(Q_{0}^{-1}\left(k^{2}\right)+B\right)^{-1} A\right]^{-1} .
$$

3. Finally, let us consider an operator of Neumann type $H_{\mathrm{N}}^{L}$ (as in Example (4) of Section 2). Now

$$
L=\left[\begin{array}{cc}
B & 0 \\
-A^{*}-I
\end{array}\right], \quad M=\left[\begin{array}{cc}
I & -A \\
0 & C
\end{array}\right],
$$

and we get a simple expression for $\Sigma(k)$, which is similar to (60):

$$
\Sigma(k)=\left[i k I+C+A^{*}\left(Q_{0}\left(k^{2}\right)-B\right)^{-1} A\right]\left[i k I-C-A^{*}\left(Q_{0}\left(k^{2}\right)-B\right)^{-1} A\right]^{-1} .
$$

In particular, if the matrix $A$ is invertible and $C$ is a Hermitian scalar matrix $(C=\gamma I)$, then

$$
\begin{aligned}
\Sigma(k)= & {\left[(i k+\gamma) A^{-1}\left(Q_{0}\left(k^{2}\right)-B\right) A^{*-1}+I\right]\left[(i k-\gamma) A^{-1}\left(Q_{0}\left(k^{2}\right)-B\right) A^{*-1}-I\right]^{-1}=} \\
& A^{-1}\left[(i k+\gamma)\left(Q_{0}\left(k^{2}\right)-B\right)+A A^{*}\right]\left[(i k-\gamma)\left(Q_{0}\left(k^{2}\right)-B\right)-A A^{*}\right]^{-1} A
\end{aligned}
$$

Remark 6. There is another way to get (70) which is similar to the derivation of (60). Namely, if we use (33) to express the resolvent of $H_{\mathrm{N}}^{L}$ and start with the function

$$
\psi_{j}(x)=\exp (i k x)-\exp (-i k x)
$$

in the channel $\mathbb{R}_{+}^{(j)}$ (instead of the function (43)), then we get, arguing as in the proof of Theorem 5,

$$
\Sigma(k)=2 i k V_{\mathrm{D}}\left(k^{2}\right)-1,
$$

where $V_{\mathrm{D}}$ is the $n \times n$-matrix in the block representation of $\left[Q_{\mathrm{D}}(z)-\Lambda\right]^{-1}$ :

$$
\left[Q_{\mathrm{D}}(z)-\Lambda\right]^{-1}=\left[\begin{array}{ll}
N_{\mathrm{D}}(z) & W_{\mathrm{D}}(z) \\
M_{\mathrm{D}}(z) & V_{\mathrm{D}}(z)
\end{array}\right] .
$$

(Note that $Q_{\mathrm{D}}$ and $\gamma_{\mathrm{D}}$ in $(\sqrt{33})$ are given by (12), (16), (26)). From $(\sqrt{72})$ and (73) we get (70) again.

It is interesting to note that for the Schrödinger operator $H=H_{0}^{B, \theta} \oplus H_{1}^{\mathrm{N}} \oplus \ldots \oplus H_{n}^{\mathrm{N}}$ (see Example 1 from Section 2), we have $\Sigma(k)=I$ independently of $k$, i.e. we have in each channel a complete reflection without phase shift. On the other hand, if $H=H_{0}^{B, \theta} \oplus H_{1}^{\mathrm{D}} \oplus$ $\ldots \oplus H_{n}^{\mathrm{D}}$ (see Example 3 from Section 2), then $\Sigma(k)=-I$ independently of $k$, i.e. there is complete reflection in each channel with a phase shift of magnitude $\pi$. 
Remark 7. With obvious modifications, the results of this section are valid for the case $d=0$ ( $X$ is a finite set of isolated points). This case is not empty as it seems at first sight. For example, if we put in (67) $A_{1}=A_{2}=X_{1}=X_{2}=0$, then

$$
\Sigma(k)=[i k C+Z]^{-1}[i k C-Z] .
$$

This is the scattering matrix for a system of quantum wires with a single common vertex derived in 49.

\section{The cases of one and two "horns"}

We consider now the most interesting particular cases. For $n=1$ we denote $q_{1}$ as $q$ and $r_{1}$ as $r$ for simplicity. If $H$ is an operator of Dirichlet type, i.e. if $H=H_{\mathrm{D}}^{L}$ (see Example 2 of Section 2), then

$$
Q\left(k^{2}\right)-L=\left[\begin{array}{cc}
Q_{0}\left(k^{2}\right)-\beta & \alpha \\
\bar{\alpha} & i k^{-1}-\gamma
\end{array}\right],
$$

where $\alpha \in \mathbb{C}, \beta, \gamma \in \mathbb{R}$ are arbitrary. In this case $\Sigma(k)$ coincides with the reflection amplitude $r(k)$. Using (61), we get

$$
\Sigma(k)=\frac{(i \gamma k-1)\left(Q_{0}\left(k^{2}\right)-\beta\right)+i|\alpha|^{2} k}{(i \gamma k+1)\left(Q_{0}\left(k^{2}\right)-\beta\right)+i|\alpha|^{2} k} .
$$

Obviously, we have $R(k) \equiv 1$ for the reflection coefficient.

Similarly, for the operator of Neumann type $H=H_{\mathrm{N}}^{L}$ (see Example 4 of Section 2) we obtain

$$
\Sigma(k)=\frac{(i k+\gamma)\left(Q_{0}\left(k^{2}\right)-\beta\right)+|\alpha|^{2}}{(i k-\gamma)\left(Q_{0}\left(k^{2}\right)-\beta\right)-|\alpha|^{2}} .
$$

It is convenient to write

$$
\Sigma(k)=: e^{i \Phi(k)},
$$

where $\Phi(k)$ is the so-called scattering phase.

Equations (75) and (76) have interesting consequences. First we recall that a point $E \in \mathbb{R}$ is called a point level of the operator $\widetilde{H}_{0}=H_{0}^{B} \equiv H_{0}^{\beta}$, if $Q(E)-\beta=0$. The spectrum of $\widetilde{H}_{0}$ (recall that $\widetilde{H}_{0}$ is a point perturbation of $H_{0}$ ) consists of all point levels and all multiple eigenvalues of the unperturbed operator $H_{0}$.

Theorem 7. 1. Let $n=1$ and $H$ be a Schrödinger operator of Dirichlet type: $H=H_{\mathrm{D}}^{L}$. Then the following assertions hold.

(1a) $\Sigma(k)=1$ (i.e. $\Phi(k) \equiv 0 \bmod 2 \pi)$ if and only if $k^{2}$ is an energy level for the point perturbation $H_{0}^{\beta}$ of $H_{0}$.

(1b) Let, in addition, $\gamma=0$. Then $\Sigma(k)=-1$ (i.e. $\Phi(k) \equiv \pi \bmod 2 \pi)$ if and only if $k^{2} \in \sigma^{p}\left(H_{0}\right)$. Therefore, for a generic point $q \in X, \Sigma(k)=-1$ if and only if $k^{2} \in \sigma\left(H_{0}\right)$.

2. Let $n=1$ and $H$ be a Schrödinger operator of Neumann type: $H=H_{\mathrm{N}}^{L}$. Then the following assertions hold. 
(2a) $\Sigma(k)=-1$ (i.e. $\Phi(k) \equiv \pi \bmod 2 \pi)$ if and only if $k^{2}$ is an energy level for the point perturbation $H_{0}^{\beta}$ of $H_{0}$.

(2b) Let, in addition, $\gamma=0$. Then $\Sigma(k)=1$ (i.e. $\Phi(k) \equiv 0 \bmod 2 \pi)$ if and only if $k^{2} \in \sigma^{p}\left(H_{0}\right)$. Therefore, for a generic point $q \in X, \Sigma(k)=1$ if and only if $k^{2} \in \sigma\left(H_{0}\right)$.

Proof. The theorem is an immediate consequence of (75) and (76).

Theorem 7 shows that by means of an infinitely thin horn $\mathbb{R}_{+}$attached to the manifold $X$ at a point $q$ we can "hear" the positive point levels of a point perturbation of $H_{0}$ at the point $q$. Moreover, if $q$ is a generic point, we can hear the positive part of the spectrum of the Schrödinger operator $H_{0}$ on $X$. Therefore, we can think of the horn $\mathbb{R}_{+}$as a kind of quantum stethoscope.

Next we consider the case of two horns $(n=2)$ in some detail. For simplicity we shall write

$$
\widetilde{Q}\left(k^{2}\right)=Q_{0}\left(k^{2}\right)-B
$$

where $B$ is a given Hermitian $2 \times 2$-matrix. We start with the Schrödinger operator $H$ of Dirichlet type, $H=H_{\mathrm{D}}^{L}$. Let $A=\left(\alpha_{j l}\right)$ be an invertible $2 \times 2$-matrix, $C=\gamma I(\gamma \in \mathbb{R})$ a scalar $2 \times 2$-matrix. We shall denote the matrix $A A^{*}$ by $N$ :

$$
N \equiv\left[\begin{array}{cc}
\nu_{11} & \nu_{12} \\
\nu_{21} & \nu_{22}
\end{array}\right]=\left[\begin{array}{cc}
\left|\alpha_{11}\right|^{2}+\left|\alpha_{12}\right|^{2} & \alpha_{11} \bar{\alpha}_{21}+\alpha_{12} \bar{\alpha}_{22} \\
\alpha_{21} \bar{\alpha}_{11}+\alpha_{22} \bar{\alpha}_{12} & \left|\alpha_{22}\right|^{2}+\left|\alpha_{21}\right|^{2}
\end{array}\right]
$$

Further we set

$$
\begin{gathered}
\Delta(k)=\left(k^{2} \gamma-i k\right)\left(\nu_{12} \widetilde{Q}_{21}\left(k^{2}\right)+\nu_{21} \widetilde{Q}_{12}\left(k^{2}\right)-\nu_{11} \widetilde{Q}_{22}\left(k^{2}\right)-\nu_{22} \widetilde{Q}_{11}\left(k^{2}\right)\right)+ \\
(i k \gamma+1)^{2} \operatorname{det} \widetilde{Q}\left(k^{2}\right)-k^{2}|\operatorname{det} A|^{2} \\
M_{11}(k)=\left(k^{2} \gamma+i k\right)\left(\nu_{21} \widetilde{Q}_{12}\left(k^{2}\right)-\nu_{22} \widetilde{Q}_{11}\left(k^{2}\right)\right)+ \\
\left(k^{2} \gamma-i k\right)\left(\nu_{12} \widetilde{Q}_{21}\left(k^{2}\right)-\nu_{11} \widetilde{Q}_{22}\left(k^{2}\right)\right)-\left(k^{2} \gamma^{2}+1\right) \operatorname{det} \widetilde{Q}\left(k^{2}\right)-k^{2}|\operatorname{det} A|^{2} \\
M_{22}(k)=\left(k^{2} \gamma+i k\right)\left(\nu_{12} \widetilde{Q}_{21}\left(k^{2}\right)-\nu_{11} \widetilde{Q}_{22}\left(k^{2}\right)\right)+ \\
\left(k^{2} \gamma-i k\right)\left(\nu_{21} \widetilde{Q}_{12}\left(k^{2}\right)-\nu_{22} \widetilde{Q}_{11}\left(k^{2}\right)\right)-\left(k^{2} \gamma^{2}+1\right) \operatorname{det} \widetilde{Q}\left(k^{2}\right)-k^{2}|\operatorname{det} A|^{2} \\
M_{12}(k)=2 i k\left(\nu_{12} \widetilde{Q}_{11}\left(k^{2}\right)-\nu_{11} \widetilde{Q}_{12}\left(k^{2}\right)\right) \\
M_{21}(k)=2 i k\left(\nu_{21} \widetilde{Q}_{22}\left(k^{2}\right)-\nu_{22} \widetilde{Q}_{21}\left(k^{2}\right)\right) .
\end{gathered}
$$

Then we have for the elements of the scattering matrix $\Sigma(k)$ :

$$
s_{11}(k)=\frac{\alpha_{11} \alpha_{22} M_{11}(k)-\alpha_{12} \alpha_{21} M_{22}(k)+\alpha_{21} \alpha_{22} M_{12}(k)-\alpha_{11} \alpha_{12} M_{21}(k)}{\operatorname{det} A \Delta(k)},
$$




$$
\begin{gathered}
s_{22}(k)=\frac{\alpha_{11} \alpha_{22} M_{22}(k)-\alpha_{12} \alpha_{21} M_{11}(k)+\alpha_{11} \alpha_{12} M_{21}(k)-\alpha_{22} \alpha_{21} M_{12}(k)}{\operatorname{det} A \Delta(k)}, \\
s_{12}(k)=\frac{\alpha_{12} \alpha_{22}\left(M_{11}(k)-M_{22}(k)\right)+\alpha_{22}^{2} M_{12}(k)-\alpha_{12}^{2} M_{21}(k)}{\operatorname{det} A \Delta(k)} \\
s_{21}(k)=\frac{\alpha_{11} \alpha_{21}\left(M_{22}(k)-M_{11}(k)\right)+\alpha_{11}^{2} M_{21}(k)-\alpha_{21}^{2} M_{12}(k)}{\operatorname{det} A \Delta(k)} .
\end{gathered}
$$

Shortly, we have

$$
\Sigma(k)=\Delta^{-1}(k) A^{-1} M(k) A,
$$

where $M=\left(M_{j l}\right)_{j, l=1,2}$.

Similarly we can obtain the scattering matrix for an operator of Neumann type, $H=H_{\mathrm{N}}^{L}$. We assume for simplicity that the matrix $A$ is diagonal: $A=\left(\alpha_{j} \delta_{j l}\right)_{1 \leq j, l \leq n}$ with real numbers $\alpha_{j}$, and that $C$ is a scalar matrix, $C=\gamma I, \gamma \in \mathbb{R}$. In this case we set

$$
\Delta_{\mathrm{N}}(k):=-\left|\alpha_{1} \alpha_{2}\right|^{2}+(i k-\gamma)\left(\left|\alpha_{2}\right|^{2} \widetilde{Q}_{11}\left(k^{2}\right)+\left|\alpha_{1}\right|^{2} \widetilde{Q}_{22}\left(k^{2}\right)\right)-(i k-\gamma)^{2} \operatorname{det} \widetilde{Q}\left(k^{2}\right) .
$$

Then (71) yields

$$
\begin{gathered}
s_{11}(k)=\left[\left|\alpha_{1} \alpha_{2}\right|^{2}+(i k+\gamma)\left|\alpha_{2}\right|^{2} \widetilde{Q}_{11}\left(k^{2}\right)-(i k-\gamma)\left|\alpha_{1}\right|^{2} \widetilde{Q}_{22}\left(k^{2}\right)+\right. \\
\left.\left(k^{2}+\gamma^{2}\right) \operatorname{det} \widetilde{Q}\left(k^{2}\right)\right] \Delta_{\mathrm{N}}^{-1}(k) \\
s_{22}(k)=\left[\left|\alpha_{1} \alpha_{2}\right|^{2}+(i k+\gamma)\left|\alpha_{1}\right|^{2} \widetilde{Q}_{22}\left(k^{2}\right)-(i k-\gamma)\left|\alpha_{2}\right|^{2} \widetilde{Q}_{11}\left(k^{2}\right)+\right. \\
\left.\left(k^{2}+\gamma^{2}\right) \operatorname{det} \widetilde{Q}\left(k^{2}\right)\right] \Delta_{\mathrm{N}}^{-1}(k) \\
s_{12}(k)=2 i k \bar{\alpha}_{1} \alpha_{2} \widetilde{Q}_{12}\left(k^{2}\right) \Delta_{\mathrm{N}}^{-1}(k) \\
s_{21}(k)=2 i k \alpha_{1} \bar{\alpha}_{2} \widetilde{Q}_{21}\left(k^{2}\right) \Delta_{\mathrm{N}}^{-1}(k) .
\end{gathered}
$$

Remark 8. If $-H^{0}$ is the Laplace-Beltrami operator, then the scattering matrix (82) coincides (up to notation) with the one derived in [24]. Moreover, if we put in (82) $B=C=0$ and $\alpha_{1}=\alpha_{2}=\alpha$, then we get

$$
s_{21}(k)=\frac{2 i k|\alpha|^{2}\left[Q_{0}\left(k^{2}\right)\right]_{12}}{k^{2} \operatorname{det} Q_{0}\left(k^{2}\right)+i k|\alpha|^{2}\left(\left[Q_{0}\left(k^{2}\right)\right]_{11}+\left[Q_{0}\left(k^{2}\right)\right]_{22}\right)-|\alpha|^{4}} .
$$

This result was obtained by A. Kiselev 46.

Let us list some interesting consequences of (77)-(80). First consider the following permutation of the matrix elements of $A: \alpha_{11} \leftrightarrow \alpha_{12}, \alpha_{21} \leftrightarrow \alpha_{22}$. Then the elements of $\Sigma(k)$ undergo the permutation $s_{11} \leftrightarrow s_{22}, s_{12} \leftrightarrow s_{21}$. The reason of this effect is intuitively clear: the permutation $\alpha_{11} \leftrightarrow \alpha_{12}, \alpha_{21} \leftrightarrow \alpha_{22}$ means that we attach the semi-axis $\mathbb{R}_{1}^{+}$to the point $q_{2}$ in place of $q_{1}$, whereas the semi-axis $\mathbb{R}_{2}^{+}$is attached to $q_{1}$. 
Another interesting consequence is related to the conducting properties of a quantummechanical system with the configuration space $\widehat{X}$. Namely, at zero temperature the ballistic conductance $\sigma(k)$ of an electric chain consisting of two one-dimensional wires $\mathbb{R}_{+}^{(1)}$ and $\mathbb{R}_{+}^{(2)}$ attached to a mesoscopic device $X$ is given by the Landauer-Büttiker formula

$$
\sigma(k)=\frac{e^{2}}{\pi \hbar} \frac{T_{12}(k)}{R_{1}(k)}
$$

where $e$ is the electron charge, $\hbar$ is the Planck constant, and $k^{2}$ is the Fermi energy [12], [52]. For a generic point $\left(q_{1}, q_{2}\right) \in X \times X, q_{1} \neq q_{2}$ and for fixed $z_{0} \in \sigma\left(H_{0}\right)$, the function $z \mapsto \operatorname{det} \widetilde{Q}_{0}(z)$ has a pole of the second order at $z_{0}$. On the other hand, the functions $z \mapsto \widetilde{Q}_{j l}(z)$ have poles at most of the first order at the same point. Therefore, for $T_{12}(k)=$ $T_{21}(k)=\left|s_{12}(k)\right|^{2}$ we have at a generic point $\left(q_{1}, q_{2}\right) \in X \times X, q_{1} \neq q_{2}$, that $T_{12}(k)=0$ if $k^{2} \in \sigma\left(H_{0}\right)$. In other words, if $k^{2} \in \sigma\left(H_{0}\right)$, then $\sigma(k)=0$. The converse is true, e.g. for a real operator $H_{0}$ (i.e. for the operator $H_{0}$ commuting with the operator $J$ of complex conjugation: $J f=\bar{f}$ ) at least if the following conditions are satisfied: (1) the matrix $A$ is diagonal and $\alpha_{11} \alpha_{22} \neq 0 ;(2) \operatorname{Im} \beta_{12} \neq 0$. In this case $\left[Q_{0}\left(k^{2}\right)\right]_{12}$ is a real number if $k^{2} \notin \sigma\left(H_{0}\right)$, and thus we have the following proposition.

Proposition 8. Let $\sigma(k)$ be the conductance of an electric chain consisting of the "wires" $\mathbb{R}_{+}^{(1)}$ and $\mathbb{R}_{+}^{(2)}$ attached to the "device" $X$ at some generic points. Suppose that the Hamiltonian of the device $X$ is a real Schrödinger operator $H_{0}$ of the Dirichlet type. If the conditions (1) and (2) above are satisfied, then $\sigma(k)$ vanishes if and only if $k^{2}$ is an eigenvalue of $H_{0}$.

Assume now that $\operatorname{dim} X \geq 2$. If the geodesic distance $r\left(q_{1}, q_{2}\right)$ between $q_{1}$ and $q_{2}$ tends to zero, then at a fixed value of the energy $k^{2}, k^{2} \notin \sigma\left(H_{0}\right)$, the numbers $\widetilde{Q}_{11}\left(k^{2}\right)$ and $\widetilde{Q}_{22}\left(k^{2}\right)$ remain bounded, whereas $\widetilde{Q}_{12}\left(k^{2}\right)$ and $\widetilde{Q}_{21}\left(k^{2}\right)$ tend to infinity. Therefore, the conductance $\sigma(k)$ tends to zero (see (79) and (80)). This paradoxical result is intimately related to an unusual behaviour of the point perturbations of the Schrödinger operators in dimensions 2 or 3 . Namely, consider a point perturbation $H_{0}^{B}$ of $H_{0}$ supported on a two-point set $\left\{q_{1}, q_{2}\right\}$. Then in the sense of the norm-resolvent convergence, $H_{0}^{B}$ tends to the unperturbed operator $H_{0}$ as $r\left(q_{1}, q_{2}\right) \rightarrow 0$. Indeed, the above considerations imply the following assertion: If $z$ is an arbitrary element of $\rho\left(H_{0}\right)$, then

$$
\left[Q_{0}(z)-B\right]^{-1} \rightarrow 0 \quad \text { as } r\left(q_{1}, q_{2}\right) \rightarrow 0
$$

A discussion of such a property of point perturbations may be found in [17]. To overcome the dificulties arising in the limit $r\left(q_{1}, q_{2}\right) \rightarrow 0$, a renormalization procedure for boundary conditions has been used [63]. It is not our intention to discuss here this subject in detail, we restrict our consideration to some consequences of (83) for the limiting behaviour of the Schrödinger operator $H$ on $\widehat{X}$.

Applying (62) to the matrix $[Q(z)-L]^{-1}$, we get

$$
[Q(z)-L]^{-1}=\left[\begin{array}{cc}
A_{11} & A_{12} \\
A_{21} & A_{22}
\end{array}\right]
$$


where

$$
\begin{aligned}
& A_{11}=\left[I-\widetilde{Q}_{0}^{-1}(z) A\left((-z)^{-1 / 2} I-C\right)^{-1} A^{*}\right]^{-1} \widetilde{Q}_{0}^{-1}(z) \\
& A_{12}=\widetilde{Q}_{0}^{-1}(z) A\left[A^{*} \widetilde{Q}_{0}^{-1}(z) A-(-z)^{-1 / 2} I+C\right]^{-1} \\
& A_{21}=\left[A^{*} \widetilde{Q}_{0}^{-1}(z) A-(-z)^{-1 / 2} I+C\right]^{-1} A^{*} \widetilde{Q}_{0}^{-1}(z) \\
& A_{22}=\left[(-z)^{-1 / 2} I-C-A^{*} \widetilde{Q}_{0}^{-1}(z) A\right]^{-1}
\end{aligned}
$$

Now using (83) we show that as $r\left(q_{1}, q_{2}\right) \rightarrow 0$, the operator $H$ tends in the norm-resolvent sense to the direct sum $H_{0} \oplus H^{\prime}$ where $H^{\prime}$ is a point perturbation (supported in 0 ) of the free Hamiltonian $-d^{2} / d x^{2}$ on the line $\mathbb{R}$. It follows from Arnold's Lemma that in the limit $r\left(q_{1}, q_{2}\right) \rightarrow 0$, we can obtain any operator of the form $H_{0} \oplus H^{\prime}$ where $H^{\prime}$ is an arbitrary point perturbation of $-d^{2} / d x^{2}$ supported on the point 0 . In the case of the operator $H=H_{\mathrm{D}}^{L}$, the limiting scattering matrix can be obtained from (60), it has the form

$$
\Sigma_{\mathrm{D}}^{\lim }(k)=(i k C-I)(i k C+I)^{-1}
$$

Similarly, if $H=H_{\mathrm{N}}^{L}$, then for the limiting form of the scattering matrix we obtain from (70)

$$
\Sigma_{\mathrm{N}}^{\lim }(k)=(i k+C)(i k-C)^{-1}
$$

We note that in both cases $\Sigma^{\lim }(k)$ depends on the block $C$ of the matrix $L$ only.

In particular, the matrix elements of $\sum_{\mathrm{N}}^{\lim }$ have the form

$$
\begin{aligned}
& s_{j l}^{\lim }(k)=\frac{-2 i k \gamma_{j l}}{k^{2}+i k \operatorname{Tr} C-\operatorname{det} C}, \quad j \neq l ; \\
& s_{j j}^{\lim }(k)=\frac{k^{2}-i k\left(\gamma_{j j}-\gamma_{l l}\right)+\operatorname{det} C}{k^{2}+i k \operatorname{Tr} C-\operatorname{det} C}, \quad j=1,2, \quad l \neq j .
\end{aligned}
$$

Moreover, if $A=I$, then the elements of the scattering matrix $\Sigma_{\mathrm{N}}(k)$ of the initial operator $H_{\mathrm{N}}^{L}$ are the following

$$
\begin{gathered}
s_{11}(k)= \\
\frac{\left(k^{2}-i k\left(\gamma_{11}-\gamma_{22}\right)+\operatorname{det} C\right) \operatorname{det} \widetilde{Q}\left(k^{2}\right)+i k\left(\widetilde{Q}_{11}\left(k^{2}\right)-\widetilde{Q}_{22}\left(k^{2}\right)\right)+\operatorname{Tr}\left(C \widetilde{Q}\left(k^{2}\right)\right)+1}{\Delta_{1}(k)} \\
s_{22}(k)= \\
\frac{\left(k^{2}-i k\left(\gamma_{22}-\gamma_{11}\right)+\operatorname{det} C\right) \operatorname{det} \widetilde{Q}\left(k^{2}\right)+i k\left(\widetilde{Q}_{22}\left(k^{2}\right)-\widetilde{Q}_{11}\left(k^{2}\right)\right)+\operatorname{Tr}\left(C \widetilde{Q}\left(k^{2}\right)\right)+1}{\Delta_{1}(k)}, \\
s_{12}(k)=\frac{2 i k\left(\widetilde{Q}_{12}\left(k^{2}\right)-\gamma_{12} \operatorname{det} \widetilde{Q}\left(k^{2}\right)\right)}{\Delta_{1}(k)}, \\
s_{21}(k)=\frac{2 i k\left(\widetilde{Q}_{21}\left(k^{2}\right)-\gamma_{21} \operatorname{det} \widetilde{Q}\left(k^{2}\right)\right)}{\Delta_{1}(k)},
\end{gathered}
$$


where

$$
\Delta_{1}(k):=\left(k^{2}+i k \operatorname{Tr} C-\operatorname{det} C\right) \operatorname{det} \widetilde{Q}\left(k^{2}\right)+i k \operatorname{Tr} \widetilde{Q}\left(k^{2}\right)-\operatorname{Tr}\left(C \widetilde{Q}\left(k^{2}\right)\right)-1 .
$$

It is interesting to compare these elements with those for the scattering matrix of $H_{\mathrm{N}}^{L}$ in the case of an arbitrary diagonal matrix $A$ and a scalar matrix $C$ (see (82)).

An important particular case of (85) and (86) arises if we choose the matrix $C$ in the form

$$
C=\left[\begin{array}{cc}
\gamma & -\gamma \\
-\gamma & \gamma
\end{array}\right]
$$

where $\gamma \in \mathbb{R}, \gamma \neq 0$. In this case

$$
\begin{aligned}
& s_{11}^{\lim }(k)=s_{22}^{\lim }(k)=\frac{-i k \gamma^{-1}}{2-i k \gamma^{-1}}, \\
& s_{12}^{\lim }(k)=s_{21}^{\lim }(k)=\frac{2}{2-i k \gamma^{-1}} .
\end{aligned}
$$

Therefore, the limiting matrix $\Sigma^{\lim }(k)$ coincides with the scattering matrix for the $\delta^{\prime}$-perturbation of the free Schrödinger operator on the line $\mathbb{R}[2]$. There is a conjecture that the scattering on the $\delta^{\prime}$-potential can be realized geometrically [5]. Our result shows that the scattering on the $\delta^{\prime}$-perturbation can be realized with an arbitrary accuracy by means of a non-trivial geometric scattering on an arbitrary compact manifold of dimension 2 or 3.

Now we give an example of non-trivial boundary conditions such that the scattering matrix of the corresponding Schrödinger operator $H^{\Lambda}$ in the limit $r\left(q_{1}, q_{2}\right) \rightarrow 0$ (for generic points) has the form

$$
\left[\begin{array}{ll}
0 & 1 \\
1 & 0
\end{array}\right]
$$

i.e. in this limit we obtain a system with zero ballistic resistance (the condition $\operatorname{dim} X \geq 2$ is kept). Namely, let us consider the boundary conditions of the form (3) where $L$ and $M$ have the following $2 \times 2$-blocks (see (64) for notation): $X_{1}=X_{2}=0, Y=I$,

$$
Z=\left[\begin{array}{cc}
0 & 0 \\
\zeta & -\zeta
\end{array}\right], \quad A_{1}=\left[\begin{array}{cc}
\alpha_{1} & 0 \\
\alpha_{2} & 0
\end{array}\right], \quad A_{2}=\left[\begin{array}{cc}
0 & 0 \\
\widehat{\alpha}_{1} & \widehat{\alpha}_{2}
\end{array}\right], \quad C=\left[\begin{array}{ll}
\gamma & \gamma \\
0 & 0
\end{array}\right]
$$

and $B$ is an arbitrary Hermitian $2 \times 2$-matrix. It is easy to prove that conditions (65) are satisfied iff $\widehat{\alpha}_{j}=\zeta \bar{\alpha}_{j}$. In this case the scattering matrix $\Sigma(k)$ is independent of $Z, A_{2}$, and $C$; its elements have the form:

$$
\begin{gathered}
s_{11}(k)=s_{22}(k)= \\
\frac{\left|\alpha_{1}\right|^{2} \widetilde{Q}_{11}\left(k^{2}\right)+\left|\alpha_{2}\right|^{2} \widetilde{Q}_{22}\left(k^{2}\right)-\bar{\alpha}_{1} \alpha_{2} \widetilde{Q}_{12}\left(k^{2}\right)-\bar{\alpha}_{2} \alpha_{1} \widetilde{Q}_{21}\left(k^{2}\right)}{\left|\alpha_{1}\right|^{2} \widetilde{Q}_{11}\left(k^{2}\right)+\left|\alpha_{2}\right|^{2} \widetilde{Q}_{22}\left(k^{2}\right)-\bar{\alpha}_{1} \alpha_{2} \widetilde{Q}_{12}\left(k^{2}\right)-\bar{\alpha}_{2} \alpha_{1} \widetilde{Q}_{21}\left(k^{2}\right)-2 i k^{-1} \operatorname{det} \widetilde{Q}\left(k^{2}\right)} \\
s_{12}(k)=s_{21}(k)= \\
2 i k^{-1} \operatorname{det} \widetilde{Q}\left(k^{2}\right) \\
\frac{2 i k^{-1} \operatorname{det} \widetilde{Q}\left(k^{2}\right)-\left|\alpha_{1}\right|^{2} \widetilde{Q}_{11}\left(k^{2}\right)-\left|\alpha_{2}\right|^{2} \widetilde{Q}_{22}\left(k^{2}\right)+\bar{\alpha}_{1} \alpha_{2} \widetilde{Q}_{12}\left(k^{2}\right)+\bar{\alpha}_{2} \alpha_{1} \widetilde{Q}_{21}\left(k^{2}\right)}{} .
\end{gathered}
$$


It is curious that the conductance of a system with the Hamiltonian $H^{\Lambda}$ is in some sense reciprocal to the one described in Proposition 8. In fact, (87) and (88) show immediately that the following proposition is true.

Proposition 9. Suppose that the semi-axes $\mathbb{R}_{+}^{(1)}$ and $\mathbb{R}_{+}^{(2)}$ are attached to $X$ in generic points and that the Schrödinger operator $H^{\Lambda}$ on $\widehat{X}$ is given as above. Then $\sigma(k)=\infty$ (i.e. the system $\widehat{X}$ is a superconductor at the energy level $k^{2}$ ) if and only if $k^{2} \in \sigma\left(H_{0}\right)$. Moreover, $\sigma(k)=0$ if and only if $k^{2}$ is an energy level for the point perturbation $H_{0}^{B}$ of $H_{0}$.

\section{A few examples}

Here some examples of Schrödinger operators $H_{0}$ on a compact manifold $X$ of constant curvature are collected for which we can give an explicit form of the Q-matrix $Q_{0}$ and, hence, get an explicit expression for the scattering matrix $\Sigma(k)$ via (60) or (70). Recall that for $j \neq l$

$$
\left[Q_{0}(z)\right]_{j l}=G_{0}\left(q_{j}, q_{l} ; z\right),
$$

where $G_{0}(x, y ; z)$ is the Green function of $H_{0}$. Therefore, as a rule, only the diagonal terms $\left.Q_{0}(z)\right]_{j j}$ are written explicitly below.

(1) Ring $\mathbb{S}_{a}$

Let $X$ be a ring $\mathbb{S}_{a}$ (i.e. a circle) of radius $a$. It is easy to show that the Green function for the Schrödinger operator of a free charged particle

$$
H_{0}=-\frac{1}{a^{2}} \frac{d^{2}}{d \varphi^{2}}
$$

$\left(\varphi \in[0,2 \pi)\right.$ being the polar coordinate on $\left.\mathbb{S}_{a}\right)$ has the form

$$
G_{0}\left(\varphi, \varphi^{\prime} ; z\right)=-\frac{1}{2 \sqrt{z}} \frac{\cos a \sqrt{z}\left(\varphi^{\prime}-\varphi \pm \pi\right)}{\sin \pi a \sqrt{z}}
$$

where the sign "plus" is taken if $\varphi \geq \varphi^{\prime}$, otherwise we take "minus". The diagonal elements of the matrix $Q_{0}$ have the form:

$$
\left[Q_{0}(z)\right]_{j j}=G_{0}\left(q_{j}, q_{j} ; z\right)
$$

(2) Aharonov-Bohm ring

Consider a ring $\mathbb{S}_{a}$ of radius $a$ located in an axially symmetric magnetic field perpendicular to the plane of the ring. Let $\Phi$ be the total magnetic flux through the ring. Put $\vartheta=\Phi / \Phi_{0}$ where $\Phi_{0}$ is the quantum of the magnetic flux: $\Phi_{0}=2 \pi \hbar c /|e|$. Then the Schrödinger operator for a charged particle in the system considered has the form

$$
H_{0}=\frac{1}{a^{2}}\left(-i \frac{d}{d \varphi}+\vartheta\right)^{2} .
$$


For the Green function we have [31]:

$$
G_{0}\left(\varphi, \varphi^{\prime} ; z\right)=\frac{1}{4 \sqrt{z}}\left[\frac{\exp \left(i\left(\varphi^{\prime}-\varphi \pm \pi\right)(\vartheta-a \sqrt{z})\right)}{\sin \pi(\vartheta-a \sqrt{z})}-\frac{\exp \left(i\left(\varphi^{\prime}-\varphi \pm \pi\right)(\vartheta+a \sqrt{z})\right)}{\sin \pi(\vartheta+a \sqrt{z})}\right]
$$

(the choice of the signs is as in the previous example). In the considered case the matrix $Q_{0}$ is given by (89) and (90) again.

(3) Flat torus $\mathbb{T}^{d}(d=2$ or 3$)$

Let $\Lambda_{d}$ be a lattice in $\mathbb{R}^{d}$ with generators $\vec{a}_{1}, \ldots, \vec{a}_{d}$ :

$$
\Lambda_{d}=\left\{n_{1} \vec{a}_{1}+\ldots+n_{d} \vec{a}_{d}: n_{j} \in \mathbb{Z}, j=1, \ldots, d\right\},
$$

and let $\Gamma_{d}$ be the dual lattice for $\Lambda_{d}$, i.e. $\Gamma_{d}$ be the lattice with generators $\vec{b}_{1}, \ldots, \vec{b}_{d}$ obeying the condition $\vec{a}_{j} \vec{b}_{k}=2 \pi \delta_{j k}$. Denote by $F_{d}$ the elementary cell for $\Lambda_{d}$ :

$$
F_{d}=\left\{x_{1} \vec{a}_{1}+\ldots+x_{d} \vec{a}_{d}:-\frac{1}{2} \leq x_{j}<\frac{1}{2}\right\}
$$

and fix points $q_{1}, \ldots, q_{n}$ from $F_{d}$. Let $H_{0}=-\Delta_{X}$ where $X$ is the the torus $\mathbb{T}^{d}=\mathbb{R}^{d} / \Lambda_{d}$. Choosing points $q_{1}, \ldots, q_{n} \in \mathbb{T}^{d}$, we have [2]:

$$
\left[Q_{0}(z)\right]_{j l}= \begin{cases}v_{d}^{-1} \lim _{\omega \rightarrow \infty} \sum_{\gamma \in \Gamma_{d},|\gamma| \leq \omega} \frac{e^{i \gamma\left(q_{j}-q_{l}\right)}}{|\gamma|^{2}-z}, & \text { if } j \neq l ; \\ (2 \pi)^{-d} \lim _{\omega \rightarrow \infty}\left[\sum_{\gamma \in \Gamma_{d},|\gamma| \leq \omega} \frac{\widehat{v}_{d}}{|\gamma|^{2}-z}-\xi_{d}(\omega)\right], & \text { if } j=l .\end{cases}
$$

Here $v_{d}$ and $\widehat{v}_{d}$ are the volumes of the tori $\mathbb{R}^{d} / \Lambda^{d}$ and $\mathbb{R}^{d} / \Gamma^{d}$ respectively; the functions $\xi_{d}$ $(d=2,3)$ have the form

$$
\xi_{d}(\omega)= \begin{cases}2 \pi \ln \omega, & \text { if } d=2 \\ 4 \pi \omega, & \text { if } d=3 .\end{cases}
$$

Using either the eigenfunction expansion for the Laplace operator on $\mathbb{T}^{d}$ or the Poisson summation formula we can get an convergent absolutely series expansion for $Q_{0}\left(k^{2}\right)_{j l}$ (see [45] for the case $d=3$ ):

$$
\left[Q_{0}\left(k^{2}\right)\right]_{j l}=(1+z) v_{d}^{-1} \sum_{\gamma \in \Gamma_{d}} \frac{e^{i \gamma\left(q_{j}-q_{l}\right)}}{\left(|\gamma|^{2}-z\right)\left(|\gamma|^{2}+1\right)}+\kappa_{d}\left(q_{j}-q_{l}\right) .
$$

Here the functions $\kappa_{d}$ are defined as follows: If $d=2$, then

$$
\kappa_{2}(x)= \begin{cases}\frac{1}{2 \pi} \sum_{\lambda \in \Lambda_{d}} K_{0}(|x+\lambda|), & \text { if } x \notin \Lambda_{d} ; \\ \frac{1}{2 \pi}\left[\sum_{\lambda \in \Lambda_{d}, \lambda \neq 0} K_{0}(|\lambda|)+\ln 2-C_{E}\right], & \text { if } x \in \Lambda_{d},\end{cases}
$$


where $K_{0}$ is the Macdonald function (i.e. the modified Bessel function of the third kind) and $C_{E}$ is the Euler constant. In the case $d=3$ we have

$$
\kappa_{3}(x)= \begin{cases}\frac{1}{4 \pi} \sum_{\lambda \in \Lambda_{d}} \frac{e^{-|x+\lambda|}}{|x+\lambda|}, & \text { if } x \notin \Lambda_{d} \\ \frac{1}{4 \pi}\left[\sum_{\lambda \in \Lambda_{d}, \lambda \neq 0} \frac{e^{-|\lambda|}}{|\lambda|}-1\right], & \text { if } x \in \Lambda_{d} .\end{cases}
$$

(4) Flat torus with Aharonov-Bohm fluxes

Consider the torus $\mathbb{T}^{d}$ as the product of $d$ Aharonov-Bohm rings $\mathbb{S}_{a_{j}}$ with fluxes $\vartheta_{j}$ $(j=1, \ldots, d)$. Let

$$
H_{j}=\frac{1}{a_{j}^{2}}\left(-i \frac{d}{d \varphi}+\vartheta_{j}\right)^{2}
$$

and

$$
H_{0}= \begin{cases}H_{1} \otimes I_{2}+I_{1} \otimes H_{2}, & \text { if } d=2 ; \\ H_{1} \otimes I_{2} \otimes I_{3}+I_{1} \otimes H_{2} \otimes I_{3}+I_{1} \otimes I_{2} \otimes H_{3}, & \text { if } d=3 .\end{cases}
$$

The operator $H_{0}$ may be considered as the Schrödinger operator on a torus $\mathbb{T}^{d}$ with a nonuniform magnetic field. Denote by $\vartheta$ the vector $\left(\vartheta_{1}, \ldots, \vartheta_{d}\right)$, then the $Q$-function $Q_{0}$ now takes the form

$$
\left[Q_{0}(z)\right]_{j l}= \begin{cases}v_{d}^{-1} \lim _{\omega \rightarrow \infty} \sum_{\gamma \in \Gamma_{d},|\gamma+\theta| \leq \omega} \frac{e^{i(\gamma+\vartheta)\left(q_{j}-q_{l}\right)}}{|\gamma+\vartheta|^{2}-z}, & \text { if } j \neq l ; \\ (2 \pi)^{-d} \lim _{\omega \rightarrow \infty}\left[\sum_{\gamma \in \Gamma_{d},|\gamma+\theta| \leq \omega} \frac{\widehat{v}_{d}}{|\gamma+\vartheta|^{2}-z}-\xi_{d}(\omega)\right], & \text { if } j=l,\end{cases}
$$

or

$$
\left[Q_{0}(z)\right]_{j l}=(1+z) v_{d}^{-1} \sum_{\gamma \in \Gamma_{d}} \frac{e^{i(\gamma+\vartheta)\left(q_{j}-q_{l}\right)}}{\left(|\gamma+\vartheta|^{2}-z\right)\left(|\gamma+\vartheta|^{2}+1\right)}+\kappa_{d, \vartheta}\left(q_{j}-q_{l}\right)
$$

Now the functions $\kappa_{d, \vartheta}(d=2,3)$ are defined as follows:

$$
\kappa_{2, \vartheta}(x)= \begin{cases}\frac{1}{2 \pi} \sum_{\lambda \in \Lambda_{d}} K_{0}(|x+\lambda|) e^{-i \vartheta \lambda}, & \text { if } x \notin \Lambda_{d} \\ \frac{1}{2 \pi}\left[\sum_{\lambda \in \Lambda_{d}, \lambda \neq 0} K_{0}(|\lambda|) e^{-i \vartheta \lambda}+\ln 2-C_{E}\right], & \text { if } x \in \Lambda_{d}\end{cases}
$$




$$
\kappa_{3, \vartheta}(x)= \begin{cases}\frac{1}{4 \pi} \sum_{\lambda \in \Lambda_{d}} \frac{e^{-|x+\lambda|-i \vartheta \lambda}}{|x+\lambda|}, & \text { if } x \notin \Lambda_{d} ; \\ \frac{1}{4 \pi}\left[\sum_{\lambda \in \Lambda_{d}, \lambda \neq 0} \frac{e^{-|\lambda|-i \vartheta \lambda}}{|\lambda|}-1\right], & \text { if } x \in \Lambda_{d} .\end{cases}
$$

(5) Flat torus $\mathbb{T}^{2}$ with a perpendicular uniform magnetic field

Consider the Euclidean plane $\mathbb{R}^{2}$ with the lattice $\Lambda_{2}$ and let $\mathbf{B}$ be a uniform magnetic field that is perpendicular to the plane and has the strength $B$. Denote by $\vartheta$ the number of the magnetic flux quanta through the elementary cell $F_{2}: \vartheta=B v_{d} / \Phi_{0}$. The Green function $G^{0}$ for the Schrödinger operator of a charged particle on the plane $\mathbb{R}^{2}$ with the field $\mathbf{B}$ has the form:

$$
\begin{gathered}
G^{0}(x, y ; z)=\frac{1}{4 \pi} \Gamma\left(\frac{1}{2}-\frac{v_{d} z}{4 \pi|\vartheta|}\right) \times \\
\exp \left[-i \pi \vartheta v_{d}^{-1} x \wedge y-\frac{\pi|\vartheta|}{2 v_{d}}(x-y)^{2}\right] \Psi\left(\frac{1}{2}-\frac{v_{d} z}{4 \pi|\vartheta|}, 1 ; \frac{\pi|\vartheta|}{v_{d}}(x-y)^{2}\right),
\end{gathered}
$$

where $\Gamma(z)$ is the Euler $\Gamma$-function, $\Psi(a, c ; z)$ is the Tricomi function (the confluent hypergeometric function), and $x \wedge y=x_{1} y_{2}-x_{2} y_{1}$ is the standard symplectic product in $\mathbb{R}^{2}$. Let the following quantization condition be satisfied: the number $\vartheta=B v_{d} / \Phi_{0}$ of the flux quanta through the cell $F_{2}$ is an integer. Then we can consider the corresponding magnetic Schrödinger operator on the torus $\mathbb{T}^{2}$. Using results from [28] we obtain for the Krein Q-matrix:

$$
\left[Q_{0}(z)\right]_{j l}=\sum_{\lambda \in \Lambda_{2}, \lambda \neq 0} G^{0}\left(\lambda+q_{j}, q_{l} ; z\right) \exp \left[\pi i \vartheta v_{d}^{-1}\left(q_{j} \wedge \lambda\right)-\pi i \vartheta \lambda_{1} \lambda_{2}\right]+\xi_{j l}(z)
$$

Here

$$
\xi_{j l}(z)= \begin{cases}G^{0}\left(q_{j}, q_{l} ; z\right), & \text { if } j \neq l \\ -\frac{1}{4 \pi}\left[\psi\left(\frac{1}{2}-\frac{v_{d} z}{4 \pi|\vartheta|}\right)+\ln \left(\pi|\vartheta| v_{d}^{-1}\right)+2 C_{E}\right], & \text { if } j=l\end{cases}
$$

where $\psi(z)$ is the digamma function (the logarithmic derivative of the $\Gamma$-function). Note that in (93), $\lambda_{1}, \lambda_{2}$ are the coordinates of $\lambda$ in the basis $\vec{a}_{1}, \vec{a}_{2}$ of $\Lambda_{2}: \lambda=\lambda_{1} \vec{a}_{1}+\lambda_{2} \vec{a}_{2}$.

(6) Sphere $\mathbb{S}_{a}^{2}$

Let $X$ be a two-dimensional sphere $\mathbb{S}_{a}^{2}$ of radius $a$, then the Green function for the Schrödinger operator $H_{0}$ of a free particle on $X, H_{0}=-\Delta_{X}$, has the form [37]:

$$
G_{0}(x, y ; z)=-\frac{1}{4 \cos (\pi t(z))} \mathcal{P}_{-\frac{1}{2}+t(z)}\left(-\cos \frac{r(x, y)}{a}\right),
$$

where $\mathcal{P}_{a}(z)$ is the Legendre function and

$$
t(z)=\frac{1}{2} \sqrt{1+4 a^{2} z} .
$$


Therefore, for every $j$

$$
\begin{gathered}
{\left[Q_{0}(z)\right]_{j j}=-\frac{1}{4 \pi}\left[\psi\left(\frac{1}{2}+t(z)\right)+\psi\left(\frac{1}{2}-t(z)\right)-2 \ln (2 a)+2 C_{E}\right]=} \\
-\frac{1}{2 \pi}\left[\psi\left(\frac{1}{2}+t(z)\right)-\frac{\pi}{2} \operatorname{tg}(\pi t(z))-\ln (2 a)+C_{E}\right] .
\end{gathered}
$$

(7) Sphere $\mathbb{S}_{a}^{3}$

Consider now a three-dimensional sphere $X=\mathbb{S}_{a}^{3}$ of radius $a$. Then the Green function for the Schrödinger operator $H_{0}$ of a free particle on $X, H_{0}=-\Delta_{X}$, reads [37]:

$$
G_{0}(x, y ; z)=\frac{1}{4 \pi a \sin \frac{r(x, y)}{a}}\left[\cos \frac{r(x, y) \sqrt{a^{2} z+1}}{a}-\sin \frac{r(x, y) \sqrt{a^{2} z+1}}{a} \operatorname{ctg} \pi \sqrt{a^{2} z+1}\right] .
$$

Therefore, for every $j$

$$
\left[Q_{0}(z)\right]_{j j}=-\frac{\sqrt{a^{2} z+1}}{4 \pi a} \operatorname{ctg} \pi \sqrt{a^{2} z+1} .
$$

(8) Compact manifold of constant negative curvature

Let now $X$ be a compact $d$-dimensional manifold of constant negative curvature (with sectional curvature $-a^{-2}$ for some $a>0$ ). We shall consider $X$ as a quotient $\mathbf{H}^{d} / \Gamma$, where $\mathbf{H}^{d}$ is the $d$-dimensional Lobachevsky space (i.e. the complete simply connected $d$ dimensional Riemannian manifold of constant negative curvature) and $\Gamma$ is a cocompact discontinuous group of motions in $\mathbf{H}^{d}$. Denote by $G_{d}^{0}$ the Green function for the LaplaceBeltrami operator on $\mathbf{H}^{d}$. Recall that

$$
\begin{gathered}
G_{d}^{0}(x, y ; z)= \\
\begin{cases}\frac{\Gamma^{2}\left(s_{2}(z)\right)}{4 \pi \Gamma\left(2 s_{2}(z)\right)}\left[\cosh \frac{r(x, y)}{2 a}\right]^{-2 s_{2}(z)} F\left(s_{2}(z), s_{2}(z) ; 2 s_{2}(z) ; \cosh ^{-2} \frac{r(x, y)}{2 a}\right), & \text { if } d=2 ; \\
\frac{\exp \left[a^{-1} r(x, y)\left(1-s_{3}(z)\right)\right]}{4 \pi a \sinh \left(a^{-1} r(x, y)\right)}, & \text { if } d=3\end{cases}
\end{gathered}
$$

(see [19], 20]). Here $F(a, b ; c ; z)$ is the Gauss hypergeometric function and

$$
s_{d}(z)=\frac{d-1+\sqrt{(d-1)^{2}-4 a^{2} z}}{2}, \quad d=2,3 .
$$

Let $H_{0}$ be a Schrödinger operator on $X$ of the form $H_{0}=-\Delta_{X}$. If $\operatorname{Re} s_{d}(z)$ is sufficiently large, then there is an expansion of the Green function $G_{0}(x, y ; z)$ for $x \neq y$ into an absolutely convergent series [25], [19], [20]:

$$
G_{0}(x, y ; z)=\sum_{\gamma \in \Gamma} G_{d}^{0}(x, \gamma y ; z) .
$$


To find $G_{0}(x, y ; z)$ for an arbitrary $z \in \mathbb{C} \backslash \sigma\left(H_{0}\right)$ we choose a number $z^{\prime}=$ Re $z+i k$, where $k \in \mathbb{R}$ is so large that the series (95) absolutely converges at $z=z^{\prime}$. Then the Neumann series

$$
R_{0}(z)=\sum_{n=0}^{\infty}\left(z-z^{\prime}\right)^{n} R_{0}^{n+1}\left(z^{\prime}\right)
$$

gives the desired value $R_{0}(z)$ and $G_{0}(x, y ; z)$ may be found as an infinite sum of iterated integral kernels $G_{0}\left(x, y ; z^{\prime}\right)$.

To find the Krein Q-function we use (95) again. If $\operatorname{Re} s_{d}(z)$ is sufficiently large, then

$$
\left[Q_{0}(z)\right]_{j j}=\sum_{\gamma \in \Gamma, \gamma \neq 1} G_{d}^{0}\left(q_{j}, \gamma q_{j} ; z\right)+\kappa_{d}(z)
$$

where

$$
\kappa_{d}(z)= \begin{cases}-\frac{1}{2 \pi}\left[\psi\left(s_{2}(z)\right)-\ln 2 a+C_{E}\right], & \text { if } d=2 ; \\ -\frac{1}{4 \pi a} \sqrt{1-a^{2} z}, & \text { if } d=3 .\end{cases}
$$

To find $\left[Q_{0}(z)\right]_{j j}$ at an arbitrary point $z, z \notin \sigma\left(H_{0}\right)$, we fix $z_{0} \in \mathbb{R}, z_{0}<0$ such that $\left[Q_{0}\left(z_{0}\right)\right]_{j j}$ is given by (96). Using the Hilbert resolvent identity and taking into consideration that the integral kernel for $R_{0}(z) R_{0}\left(z_{0}\right)$ is continuous [19], [20], we get:

$$
\left[Q_{0}(z)\right]_{j j}=\left[Q_{0}\left(z_{0}\right)\right]_{j j}+\left(z-z_{0}\right) \int_{X} G_{d}^{0}\left(q_{j}, x ; z\right) G_{d}^{0}\left(x, q_{j} ; z_{0}\right) d \lambda(x) .
$$

Remark 9. In some sense (96) is an analogue of (91) for the space of constant negative curvature. Let us consider for simplicity the case of one horn $\left(n=1, q_{1}=q\right)$ and try to transform (96) to an equality similar to (92) hoping to get a more convenient expression. First note that in general $Q_{0}(z)$ depends on $q$ : $Q_{0}(z)=Q_{0}(z, q)$. But the Poisson summation formula gives us an averaged value $Q_{0}^{\text {av }}(z)$ of $Q_{0}(z, q)$ :

$$
Q_{0}^{\mathrm{av}}(z):=(\operatorname{vol} X)^{-1} \int_{X} Q_{0}(z, q) d q
$$

If $X$ is a homogeneous manifold, then $Q_{0}^{\mathrm{av}}(z)$ is independent of $q$ and $Q_{0}(z)=Q_{0}^{\mathrm{av}}(z)$. Therefore, in the case of the torus $\mathbb{T}^{d}, Q_{0}(z)$ is given by (92). Let now $X$ be a compact surface of constant negative curvature. In this case the role of the Poisson summation formula is played by the Selberg trace formula. Using the Selberg formula in the form obtained by P. Cartier and A. Voros [13 we get an explicit expression for $Q_{0}^{\text {av }}(z)$ up to an additive constant $c$ :

$$
Q_{0}^{\mathrm{av}}(z)=(2-2 g) \psi\left(s_{2}(z)\right)+\frac{1}{\sqrt{1-4 a^{2} z}} \frac{z_{X}^{\prime}\left(s_{2}(z)\right)}{z_{X}\left(s_{2}(z)\right)}+c,
$$

where $g$ is the genus of $X, z_{X}(s)$ is the Selberg zeta function for $X$ [43], [67], and $s_{2}(z)$ is given by (94). Note that without loss of generality we can put $c=0$, otherwise we add $c$ to the parameter $\beta$ in (75) and (76). 
(9) Compact Riemann surface of constant negative curvature with a uniform magnetic field

Consider the Lobachevsky plane $\mathbf{H}^{2}$ with a uniform magnetic field $\mathbf{B}$ of strength $B$ perpendicular to the plane [15]. Using the Poincaré half-plane realization for $\mathbf{H}^{2}\left(\mathbf{H}^{2}=\{x \in\right.$ $\left.\mathbb{R}^{2}: x_{2}>0\right\}$ with the metric $r(x, y)=a \cosh ^{-1}\left(1+\left(2 x_{2} y_{2}\right)^{-1}|x-y|^{2}\right)$ ), we have the following representation for the Green function $G^{0}(x, y ; z)$ of the magnetic Schrödinger operator on $\mathbf{H}^{2}$ [19], 15]:

$$
\begin{gathered}
G^{0}(x, y ; z)=\frac{\exp (i b \varphi)}{4 \pi} \frac{\Gamma(t(z)+b) \Gamma(t(z)-b)}{\Gamma(2 t(z))} \times \\
{\left[\cosh \frac{r(x, y)}{2 a}\right]^{-2 t(z)} F\left(t(z)+b, t(z)-b ; 2 t(z) ; \cosh ^{-2} \frac{r(x, y)}{2 a}\right),}
\end{gathered}
$$

where

$$
\begin{gathered}
\varphi=2 \operatorname{arctg} \frac{x_{1}-y_{1}}{x_{2}+y_{2}}, \\
t(z)=\frac{1}{2}\left(1+\sqrt{1-4\left(a^{2} z-b^{2}\right)}\right), \\
b=B a^{2} / \Phi_{0} .
\end{gathered}
$$

Let $S_{\Gamma}$ be the area of a fundamental domain for $\Gamma$ and suppose that $B S_{\Gamma} / \Phi_{0}$ is an integer. Then one can define the magnetic Schrödinger operator $H_{0}$ on the manifold $X=\mathbf{H}^{2} / \Gamma$, and its Green function has the form (95) for sufficiently large $\operatorname{Re} t(z)$ [19]. For this $t(z)$ we obtain, using a result from [8],

$$
\left[Q_{0}(z)\right]_{j l}=\sum_{\gamma \in \Gamma, \gamma \neq 1} G^{0}\left(q_{j}, \gamma q_{l} ; z\right)+\xi_{j l}(z)
$$

where

$$
\xi_{j l}(z)= \begin{cases}G^{0}\left(q_{j}, q_{l} ; z\right), & \text { if } j \neq l \\ -\frac{1}{4 \pi}\left[\psi(t(z)+b)-\psi(t(z)-b)-2 \ln 2 a+2 C_{E}\right], & \text { if } j=l\end{cases}
$$

To define the Q-matrix at other points of $\mathbb{C} \backslash \sigma\left(H_{0}\right)$ it is sufficient to apply the method presented in Example 7.

\section{Acknowledgments}

The authors are grateful to P. Exner for useful discussions and for the possibility to get acquainted with the results of [24] before publication.

We gratefully acknowledge grants of DFG (No. 436 RUS 113/572/1) and INTAS (No. 00-257). The second named author also very grateful to RFBR (Grant No. 02-0100804) and to the SFB 288 for a financial support, and to Humboldt University of Berlin for warm hospitality during the preparation of this paper. 


\section{References}

[1] E. Akkermans, A. Comtet, J. Desbois, G. Montambaux, Ch. Texier. Spectral determinant on quantum graphs. Ann. Phys. 284 (2000), 10-51.

[2] S. Albeverio, F. Gesztesy, R. Høegh-Krohn, and H. Holden. Solvable models in quantum mechanics. Springer-Verlag: Berlin etc., 1988.

[3] V. I. Arnold. On a characteristic class entering into the quantization condition (in Russian). Funk. Anal. i Prilozh. 1, No 1 (1967), 1-14; Engl. transl.: Funkt. Anal. Appl. 1, No 1 (1967), 1-13.

[4] I. G. Avramidi. Green functions of higher-order differential operators. J. Math. Phys. 39 (1998), 2889-2909.

[5] J. E. Avron, P. Exner, Y. Last. Periodic Schrödinger operators with large gaps and Wannier-Stark ladders. Phys. Rev. Lett. 72 (1994), 896-899.

[6] V. Bogevolnov, A. Mikhailova, B. Pavlov, A. Yafyasov. About scattering on the ring. University of Auckland, Dep. of Math. Report Series, No. 413 (1999), 16 pp.

[7] N. Bourbaki. Algèbre. Ch. IX. Herman: Paris, 1958.

[8] J. Brüning, V. A. Geyler. Gauge periodic point perturbations on the Lobachevsky plane (in Russian). Teor. i Mat. Fiz. 119 (1999), 363-380; Engl. transl.: Theor. Math. Phys. 119 (1999), 687-697.

[9] J. Brüning, V. A. Geyler. The spectrum of periodic point perturbations and the Krein resolvent formula. Oper. Theory: Adv. and Appl. 117 (2000), 71-86.

[10] J. Brüning, V. A. Geyler. Limiting absorption principle and the particle current conservation for one-dimensional geometric scattering. Proc. Intern. Sem. "Day on Diffraction in New Millennium." St.-Petersburg, 2001, 87-96.

[11] J. Brüning, V. A. Geyler, M. A. Margulis, M. A. Pyataev. Ballistic conductance of a quantum sphere. J. Phys. A: Math. Gen. 35 (2002), 4239-4247.

[12] M. Büttiker, Y. Imry, R. Landauer, S. Pinhas. Generalized many-channel conductance formula with applications to small rings. Phys. Rev. B31 (1985), 6207-6212.

[13] P. Cartier, A. Voros. Une nouvelle interprétation de formule des traces de Selberg. In.: The Grothendieck Festschrift./ Eds. P. Cartier e.a. V. II. Birkhäuser: Boston etc. 1990. (Progress in Mathematics, V. 87). P. 1-66.

[14] Y. Colin de Verdiere. Pseudo-Laplaciens. I. Ann. Inst. Fourier. 32, No 3 (1982), 275-286.

[15] A. Comtet. On the Landau level on the hyperbolic plane. Ann. Phys. 173 (1987), 185209.

[16] L. Dabrowski, H. Grosse. On non local point interactions in one, two and three dimensions. J. Math. Phys. 26 (1985), 2777-2780.

[17] Y. N. Demkov, V. N. Ostrovsky. Zero-range potentials and their applications in atomic physics. Plenum Press: New York, 1988.

[18] V. A. Derkach, M. M. Malamud. Generalized resolvents and the boundary value problems for Hermitian operators with gaps. J. Funct. Anal. 95 (1991), 1-95. 
[19] J. Elstrodt. Die Resolvente zum Eigenwertproblem der automorphen Formen in der hyperbolische Ebene. I. Math. Ann. 203 (1973), 295-330; II. Math. Z. 132 (1973), 99134; III. Math. Ann. 208 (1974), 99-132.

[20] J. Elstrodt, F. Grunnewald, J. Mennicke. Groups acting on hyperbolic spaces. SpringerVerlag: Berlin etc., 1998.

[21] P. Exner, P. Šeba. Quantum motion on a half-line connected to a plane. J. Math. Phys. 28 (1987), 386-391.

[22] P. Exner, P. Šeba. Free quantum motion on a branching graph. Rep. Math. Phys. 28 (1989), 7-26.

[23] P. Exner, P. Šeba. Resonance statistics in a microwave cavity with a thin antenna. Phys. Lett. A228 (1997), 146-150.

[24] P. Exner, M. Tater, D. Vaněk. A single-mode quantum transport in serial-structure geometric scattering. J. Math. Phys. 42 (2001), 4050-4078.

[25] L. D. Faddeev. Expansion in eigenfunctions of the Laplace operator on the fundamental domain of a discrete group on the Lobačevskil plane (in Russian). Trudy Mosk. Matem. Ob-va. 17 (1967), 323-350; Engl. transl.: Trans. Moscow Math. Soc. 17 (1967), 357-386.

[26] L. D. Faddeev, B. S. Pavlov. Scattering theory and automorphic functions (in Russian). Semin. of Steklov Math. Inst. of Leningrad. 27 (1972), 161-193.

[27] N. I. Gerasimenko, B. S. Pavlov. Scattering problems on noncompact graphs (in Russian). Teor. i Mat. Fiz. 74 (1988), 345-359; Engl. transl.: Theor. Math. Phys. $\mathbf{7 4}$ (1988), 230-240.

[28] V. A. Geyler. The two-dimensional Schrödinger operators with a uniform magnetic field and its perturbation by periodic zero-range potentials (in Russian). Algebra i Analiz $\mathbf{3}$, No 3 (1991), 1-48; Engl. transl.: St.-Petersburg Math. J. 3 (1992), 489-532.

[29] V. A. Geyler, V. V. Demidov, V. A. Margulis. Transport in the two-terminal AharonovBohm ring (in Russian). Submitted to J. Techn. Phys.

[30] V. A. Geyler, V. A. Margulis, I. I. Chuchaev. Zero-range potentials and Carleman operators (in Russian). Sibir. matem. zhurn. 36 (1995), 828-841; Engl. transl.: Siberian Math. J. 36 (1995), 714-726.

[31] V. A. Geyler, A. V. Popov. Localization in a periodic system of the Aharonov-Bohm rings. Rep. Math. Phys. 42 (1998), 347-358.

[32] V. A. Geyler, I. Yu. Popov. Ballistic transport in nanostructures: Explicitly solvable model (in Russian). Teor. i Mat. Fiz. 107 (1996), 12-20; Engl. transl.: Theor. Math. Phys. 107 (1996), 427-434.

[33] V. A. Geyler, I. Yu. Popov, S. L. Popova. Transmission coefficient for ballistic transport through quantum resonator. Rep. Math. Phys. 40 (1997), 531-538.

[34] R. Godement. The decomposition of $L^{2}(G / \Gamma)$ for $\Gamma=S L(2, \mathbb{Z})$. Proc. Symp. pure Math. 9 (1966), 211-224.

[35] V. I. Gorbachuk, M. A. Gorbachuk. Boundary value problems for operator differential equations. Kluwer Acad. Publ.: Dordrecht etc., 1991. 
[36] M. Gromov. Metric structures for Riemannian and non-Riemannian spaces. Birkhäuser: Boston etc., 1999.

[37] C. Grosche, F. Steiner. Handbook of Feynman path integrals. Springer-Verlag: Berlin etc., 1998.

[38] M. C. Gutzwiller. Chaos in classical and quantum mechanics. Springer-Verlag: New York etc., 1990.

[39] M. C. Gutzwiller. Physics and Selberg's trace formula. Contemp. Math. 53 (1986), 215251.

[40] M. C. Gutzwiller. Stochastic behavior in quantum scattering. Physica D. 7 (1983), 341355 .

[41] M. Harmer. Hermitian symplectic geometry and the factorization of the scattering matrix on graphs. J. Math. A: Math. Gen. 33 (2000), 9015-9032.

[42] M. Harmer. Hermitian symplectic geometry and extension theory. J. Math. A: Math. Gen. 33 (2000), 9193-9203.

[43] D. A. Hejkal. The Selberg trace formula for $P S L(2, \mathbb{R})$. V. I. Lect. Notes Math. 548 (1976), 1-516.

[44] R. A. Horn, C. R. Johnson. Matrix analysis. Cambridge Univ. Press: Cambridge, 1986.

[45] Yu. E. Karpeshina. Spectrum and eigenfunctions of Schrödinger operator with zerorange potential of homogeneous lattice type in three-dimensional space (in Russian). Teor. i Mat. Fiz. 57 (1983), 304-313; Engl. transl.: Theor. Math. Phys. 57 (1983), 1156-1162.

[46] A. Kiselev. Some examples in one-dimensional "geometric" scattering on manifolds. $J$. Math. Anal. Appl. 212 (1997), 263-280.

[47] A. N. Kochubei. On extensions of symmetric operators and symmetric boundary relations (in Russian). Mat. Zametki. 17, No 1 (1975), 41-48; Engl. transl.: Math. Notes. 17, No 1 (1975), 25-28.

[48] A. N. Kochubei. Elliptic operators with boundary conditions on a subspaces of zero measure (in Russian). Funk. Anal. i Prilozh. 16, No 2 (1982), 74-75; Engl. transl.: Funkt. Anal. Appl. 16 (1982), 137-139.

[49] V. Kostrykin, R. Schrader. Kirchhoff's rule for quantum wires. I. J. Phys. A: Math. Gen. 32 (1999), 595-630; II. Fortschr. Phys. 48 (2000), 703-716.

[50] M. G. Krein. The theory of self-adjoint extensions of semi-bounded Hermitian operators and its applications. I (Russian). Matem. Sbornik. 20 (1947), 431-495.

[51] M. G. Krein, H. K. Langer. Defect subspace and generalized resolvents of an Hermitian operators in the space $\Pi_{\kappa}$ (in Russian). Funk. Anal. $i$ Prilozh. 5, No 2 (1971), 59-71; Engl. transl.: Funct. Anal. and Appl. 5 (1971), 217-228.

[52] R. Landauer. Electrical resistance of disordered one-dimensional lattices. Philosoph. Magazin. 21 (1970), 863-867.

[53] P. D. Lax, R. S. Phillips. Scattering theory for automorphic functions. Princeton Univ. Press: Princeton, 1976. 
[54] R. B. Melrose. Geometric scattering theory. Cambridge Univ. Press: Cambridge, 1995.

[55] A. Mikhailova, B. Pavlov. Quantum domain as a triadic relay. University of Auckland, Dep. of Math. Report Series, No. 439 (2000), 24 pp.

[56] A. Mikhailova, B. Pavlov, I. Popov, T. Rudakova, A. M. Yafyasov. Scattering on a compact domain with few semiinfinite wires attached: resonance case. University of Auckland, Dep. of Math. Report Series, No. 420 (1999), 17 pp. Submitted to Math. Nachr.

[57] C. Miranda. Equazioni alle derivate parziali di tipo ellittico. Springer-Verlag: Berlin etc., 1955.

[58] M. A. Naimark. Linear differential operators, Part II. Linear Differential operators in Hilbert space. Ungar: New York, 1968.

[59] S. P. Novikov. Schrödinger operators on graphs and symplectic geometry. Fields Institute Commun. 24 (1999), 397-413.

[60] B. S. Pavlov. The theory of extensions and explicitly solvable models (in Russian). Uspekhi Mat. Nauk 42, No 6 (1987), 99-131; Engl. transl.: Russian Math. Surv. 42, No 6 (1987), 127-168.

[61] B. S. Pavlov, I. Yu. Popov, V. A. Geyler, O. S. Pershenko. Possible construction of a quantum multiplexer. Europhys. Lett. 52 (2000), 196-202.

[62] I. Yu. Popov, V. A. Geyler, A. V. Popov. Localization in a system of the AharonovBohm rings (Russian). Fiz. Tverd. Tela. 41 (1999), 910-913; Engl. transl.: Phys. Solid State. 41 (1999), 827-829.

[63] T. K. Rebane, R. I. Sharibjanov. Magnetic properties of the simplest systems in the zero-range potential model (Russian). Theor. and Experim. Chemistry. 10 (1974), 444449.

[64] M. Reed, B. Simon. Methods of modern mathematical physics. III. Scattering theory. Acad. Press: New York etc., 1979.

[65] M. Reed, B. Simon. Methods of modern mathematical physics. IV. Analysis of operators. Acad. Press: New York etc., 1978.

[66] B.-M. Schulze. Transmission algebras on singular spaces with components of different dimensions. Operator Theory: Adv. and Appl. 78 (1995), 322-342.

[67] A. Selberg. Harmonic analysis and discontinuous groups in weakly symmetric Riemannian spaces with applications to Dirichlet series. J. Ind. Math. Soc. 20 (1956), 47-87.

[68] A. B. Venkov. Spectral theory of automorphic functions and its applications. Kluwer Acad. Publ.: Dordrecht, 1990.

[69] J. Zorbas. Perturbation of self-adjoint operators by Dirac distributions. J. Math. Phys. 21 (1980), 840-847. 